This item was submitted to Loughborough's Institutional Repository (https://dspace.lboro.ac.uk/) by the author and is made available under the following Creative Commons Licence conditions.

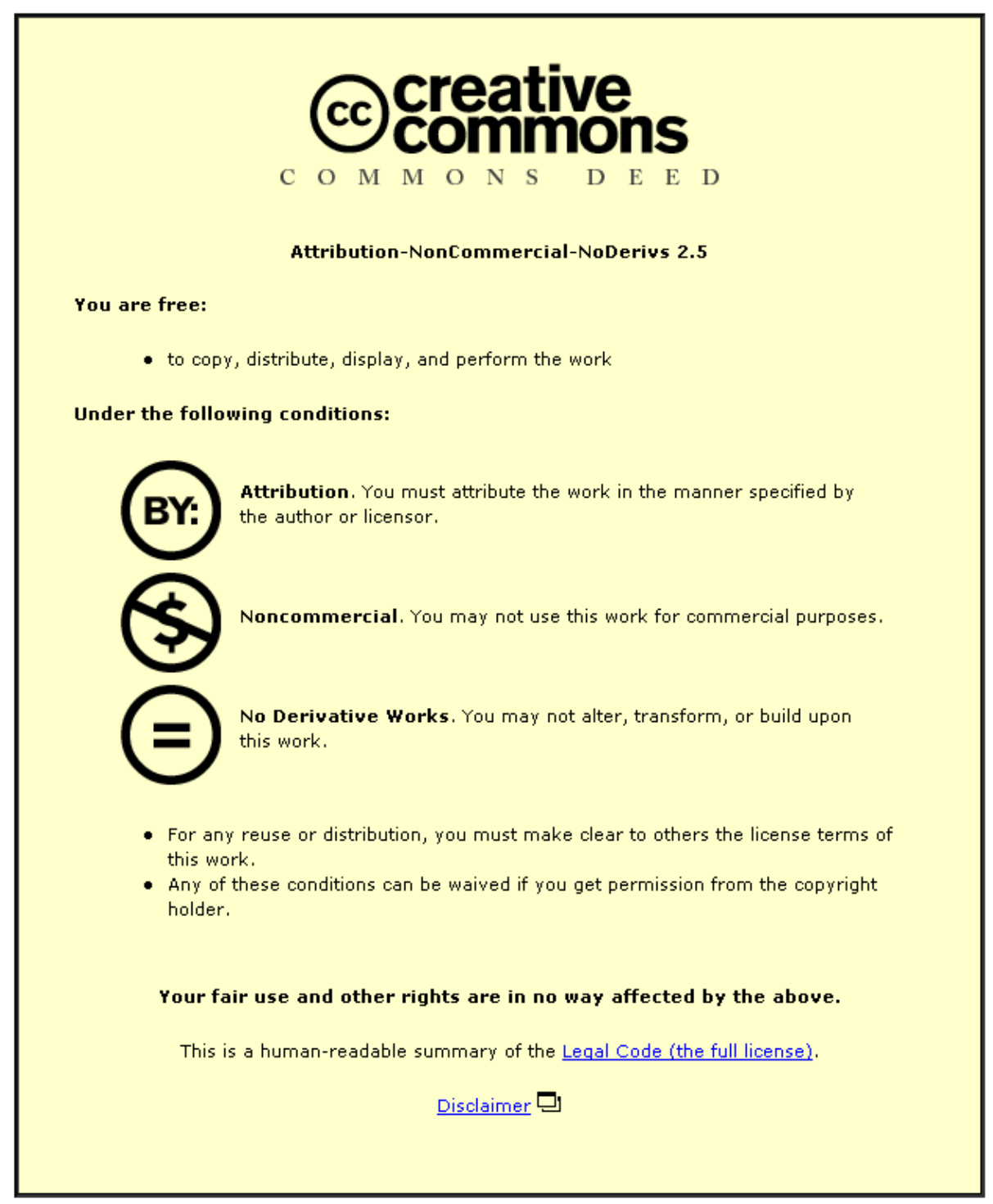

For the full text of this licence, please go to: http://creativecommons.org/licenses/by-nc-nd/2.5/ 


\title{
Reversible coagulation of colloidal suspension in shallow potential wells: direct numerical simulation
}

\author{
Nina Kovalchuk ${ }^{1,2}$, Victor Starov ${ }^{1 \mathrm{f}}$, Paul Langston ${ }^{3}$, Nidal Hilal ${ }^{3}$ \\ ${ }^{1}$ Department of Chemical Engineering, Loughborough University, Loughborough, \\ LE11 3TU (UK) \\ ${ }^{2}$ Institute of Biocolloid Chemistry, 03142 Kiev, Ukraine \\ ${ }^{3}$ School of Chemical, and Environmental Engineering, University of Nottingham, \\ University Park, Nottingham, NG7 2RD (UK)
}

\begin{abstract}
Brownian dynamics computer simulations of aggregation in 2D colloidal suspensions are discussed. The simulations are based on the Langevin equations, pairwise interaction between colloidal particles and take into account Brownian, hydrodynamic and colloidal forces. The chosen mathematical model enables to predict the correct values of diffusion coefficient of freely moving particle, the mean value of kinetic energy for each particle in ensemble of interacting colloidal particles and residence times of colloidal particles inside the potential wells of different depths. The simulations allows monitoring formation and breakage of clusters in a suspension as well as time dependence of the mean cluster size.
\end{abstract}

Key words: colloidal suspensions, Langevin equations, colloidal forces, Brownian forces, hydrodynamic interactions, fluctuation-dissipation theorem, clusters.

\footnotetext{
${ }^{f}$ Corresponding author: V.M.Starov@lboro.ac.uk
} 


\section{Introduction}

Stability of colloidal suspension is determined by the balance of repulsive and attractive forces acting between the colloidal particles [1,2]. Suspension remains kinetically stable if high enough potential barrier due to electrostatic or steric repulsion prevent coagulation in the deep primary potential well. The most interesting behaviour is however expected if the coagulation occurs in the relatively shallow potential well with depth comparable to the energy of thermal motion. In this case the structure of suspension in the simplest case of perikinetic flocculation is determined by dynamic equilibrium between aggregation due to attraction between particles and disaggregation due to their thermal motion $[3,4]$. The coagulation in the shallow potential well is reversible, and the clusters can be destroyed by an increase of temperature (an increase of the energy of thermal motion) or by a decrease of the salt concentration (a decrease of the depth of the secondary potential well), what is not the case for coagulation in the deep primary potential well.

Numerous experimental investigations have shown that the flocculation in the shallow potential well often results in formation a phase of stable clusters (clusters) of colloidal particles [5-13]. Direct observations by confocal microscope confirmed that clusters are at equilibrium with singlets and that their size remains stable during the long time [9-12]. The cluster size varies depending on interaction forces between particles and solid volume fraction of suspension. Usually clusters are observed at relatively small solid volume fractions. Increase of the solid volume fraction above certain threshold value can lead to formation of gel-like structure $[9,10,12]$.

It is noteworthy, that depending on the system studied, the mean number particles in cluster can vary in wide ranges and sometimes clusters can contain up to thousands particles [13]. It is not clear, whether such a large clusters, and even smaller, containing several tens of particles can appear as a result of reversible coagulation. That is why there is a number of different approaches explaining the existence of stable clusters. In [14] the capillarity approximation is proposed, where the clusters are treated as uniform droplets. The driving force for the cluster growth in this approach is the decrease of the surface energy of the system whereas the stabilising factor is the Coulomb repulsion. Another approach is based on the calculation of ground state energy per particle depending on the number of particles in the cluster, which according to $[15,16]$ has a minimum at certain number of particles 
in cluster. These approaches does not take into account the energy of particles thermal motion, but the only interaction energy.

Often the aggregation in suspension is considered as a phase transition from fluid composed of singlets to liquid of clusters. Then the methods of statistical thermodynamic, taking into account the contribution of entropy to the free energy of system are used to find the phase coexistence region, where the singlets are in dynamic equilibrium with clusters [17-20].

Computer simulations using Monte Carlo methods [21], population balance equations [22], Brownian dynamics methods [23,24] are also widely used in theoretical treatment of the problem. Nevertheless, despite of large amount publications in this field, the mechanisms responsible for the formation of the phase of stable clusters as well as methods to control their appearance and properties are still under discussion.

Below we present results of direct numerical simulation of aggregation in colloidal suspension performed using the Brownian Dynamic method. Note, the simulations have been performed for the two-dimensional case (monolayer of colloidal particles) and extending the results to the three-dimensional system requires additional discussion. The mathematical model used in the simulations is based on the first principles without any fitting parameters. To prevent the artificial pumping or dissipation energy in the considered system due to numerical effects, the mean kinetic energy of each particle was monitored through the simulation process. It is expected that simulation allows understanding in more details the mechanisms governing the cluster formation due to reversible flocculation in the shallow potential well and to determine the system parameters most important for this process.

\section{Mathematical model}

The model system is composed of $\mathrm{N}$ identical spherical colloidal particles moving in two dimensions. The particles motion is governed by Langevin equations [25]:

$m \frac{d V_{i}}{d t}=-\sum_{j=1}^{2 N} \varsigma_{i j} V_{j}+\sum_{j=1}^{2 N} \alpha_{i j} f_{j}+\sum_{j=1}^{2 N} F_{i j}$

where $i, j=1 \ldots 2 \mathrm{~N}, \quad m=\frac{4}{3} \pi a^{3}\left(\rho_{p}+0.5 \rho_{l}\right)$ is the mass of the particle (including the added mass), $V$ is the particle velocity, $t$ is the time, $a$ is the radius of the particle, $\rho_{p}$ 
is the density of the particle material, $\rho_{l}$ is the density of the suspending liquid, $\varsigma_{i j}$ is the element of the hydrodynamic resistance matrix, $\hat{\alpha} f$ represents the Brownian forces, with $f_{i}$ being a random quantity, normally distributed, with

$$
\begin{aligned}
& \left\langle f_{i}\right\rangle=0, \\
& \left\langle f_{i}(t) f_{j}\left(t^{\prime}\right)\right\rangle=2 \delta_{i j} \delta\left(t-t^{\prime}\right),
\end{aligned}
$$

$\boldsymbol{F}$ represents the colloidal forces.

The hydrodynamic resistance coefficients, $\varsigma_{i j}$, and $\alpha_{i j}$ detrmining the value of Brownian forces , are related according to the fluctuation-dissipation theorem [26]:

$$
\varsigma_{i j}=\frac{1}{k T} \sum_{l} \alpha_{i l} \alpha_{l j}
$$

where $k$ is the Boltzmann constant, $T$ is the absolute temperature.

All considered forces are assumed to be pairwise additive. Particles rotation is neglected. As interactions at small distances are the most crucial for aggregation/disaggregation processes, the lubrication approximation was used for calculation of the hydrodynamic interactions. Therefore it was assumed that hydrodynamic interaction between two particles depends only on distance between them and their relative velocities:

$$
\begin{aligned}
& F_{H 13}=-\varsigma_{x}\left(V_{1}-V_{3}\right), \\
& F_{H 24}=-\varsigma_{y}\left(V_{2}-V_{4}\right),
\end{aligned}
$$

where $F_{H 13}, F_{H 24}$ are the hydrodynamic force components acting on the particle 1 due to its interaction with particle 2 in the particle pair local co-ordinate, where axis $x$ is parallel to the line connecting the particle centres, $y$ is in the tangential direction. $V_{1}$ and $V_{2}$ are $x$ and $y$ velocity components of particle $1, V_{3}$ and $V_{4}$ are $x$ and $y$ velocity components of particle 2 .

At the small separation between the particles surfaces $h \leq 0.1 a$ the coefficients $\varsigma_{x}$ and $\varsigma_{y}$ are calculated according to the equations proposed in [27]:

$$
\begin{array}{ll}
\varsigma_{x}=\frac{3}{2} \pi \mu \frac{a^{2}}{h}, & \text { at } h \leq 0.1 a, \\
\varsigma_{y}=\pi \mu a \ln \left(\frac{a}{h}\right), & \text { at } h \leq 0.1 a .
\end{array}
$$

As the numerical calculation of a logarithmic function is time consuming, the following approximation of Eq. (9) is used in the simulations: 
$\varsigma_{y}=-\pi \mu a\left(1.8043+0.05 \frac{a}{h}-3 \cdot 10^{-6} \frac{a^{2}}{h^{2}}\right), \quad$ at $h \leq 0.1 a$.

The hydrodynamic interactions at separations $h>2.5 a$ are neglected and the hydrodynamic force in this case is determined by the Stokes law:

$F_{H i i}=6 \pi \mu a V_{i}$,

where $\mu$ is the dynamic viscosity of the suspending liquid.

The interaction forces for $0.1 a \leq h \leq 2.5 a$ are fitted providing a smooth transition between forces at $h \leq 0.1 a$ and 0 at $h=2.5 a$.

Taking into account Eqs. (5), (6) and (9) the matrix of hydrodynamic resistance for two interacting particles can be written as follows:

$\hat{\varsigma}=\left(\begin{array}{cccc}\varsigma+\varsigma_{x} & 0 & -\varsigma_{x} & 0 \\ 0 & \varsigma+\varsigma_{y} & 0 & -\varsigma_{y} \\ -\varsigma_{x} & 0 & \varsigma+\varsigma_{x} & 0 \\ 0 & -\varsigma_{y} & 0 & \varsigma+\varsigma_{y}\end{array}\right)$

i.e. it contains the only 4 different elements $\varsigma_{x}, \varsigma_{y}, \varsigma+\varsigma_{x}$ and $\varsigma+\varsigma_{y}$. The matrix of Brownian coefficients has the same form as the matrix of hydrodynamic coefficients:

$\hat{\alpha}=\left(\begin{array}{cccc}\alpha_{11} & 0 & \alpha_{13} & 0 \\ 0 & \alpha_{22} & 0 & \alpha_{24} \\ \alpha_{13} & 0 & \alpha_{11} & 0 \\ 0 & \alpha_{24} & 0 & \alpha_{22}\end{array}\right)$.

The coefficients $\alpha_{i j}$ are found by solving the set of Eqs (4) for elements of matrices (10) and (11):

$$
\begin{aligned}
& \alpha_{11}=\frac{1}{2} \sqrt{k T}\left(\sqrt{\varsigma}+\sqrt{\varsigma+2 \varsigma_{x}}\right) \\
& \alpha_{13}=\frac{1}{2} \sqrt{k T}\left(\sqrt{\varsigma}-\sqrt{\varsigma+2 \varsigma_{x}}\right) \\
& \alpha_{22}=\frac{1}{2} \sqrt{k T}\left(\sqrt{\varsigma}+\sqrt{\varsigma+2 \varsigma_{y}}\right) \\
& \alpha_{24}=\frac{1}{2} \sqrt{k T}\left(\sqrt{\varsigma}-\sqrt{\varsigma+2 \varsigma_{y}}\right)
\end{aligned} .
$$

The components of random forces $f_{i}$ obeying Eq. (2,3) were modelled in numerical simulations as [28, 29]:

$$
f_{i}=\sqrt{\frac{2}{d t}} R_{N D}
$$


where $d t$ is the time step used in computer simulations, $R_{N D}$ is a random number from a normal distribution with the mean value equal to zero and standard deviation equal to 1 .

The simplified expression has been adopted for the dependency of normal force per unit area between two parallel flat surfaces, $\Phi(h)$, to model the colloidal interaction between particles (Fig. 1a). However, the adopted expression represents the main features of the real force: the presence of both a repulsion and an attraction as well as the presence of a potential well. The separations between particles corresponding to the zeros of $\Phi(h), h_{1}$ and $h_{2}$, the separation corresponding to the minimum of $\Phi(h), h_{0}$, and the depth of the potential well, $U_{\min }$, have been selected as parameters to describe the colloidal interactions.

The colloidal force acting along the centre line between particles was calculated according to the Derjaguin's approximation [1, 2]:

$$
F(h)=\pi a \int_{h}^{\infty} \Phi(h) d h,
$$

Eq. (14) is valid if $h_{2}<<a$, which is the case because $a=1 \mu \mathrm{m}$ used below.

The interaction energy (Fig. 1c) was calculated as:

$U(h)=\int_{h}^{\infty} F(h) d h$

The governing equations (1) were solved by the finite difference Euler's method taking into account the interaction of a particle with nearest neighbours. Periodic boundary conditions were imposed on the whole system to simulate the behaviour of an unbounded colloidal suspension. A random as well as uniform initial distribution of particles over the 2-D lattice was used. The initial particles velocities, $V_{i}$, were generated according to the Maxwell distribution.

\section{Results and discussions}

To validate the mathematical model and numerical scheme used, the value of diffusion coefficient of single particle, the mean value of kinetic energy of each particle in ensemble and mean residence time of particle in potential well were found from the results of numerical simulations and compared with corresponding theoretical values. 
The diffusion coefficient of a single freely moving particle was calculated as [22]:

$D=\frac{\left\langle\left(x-x_{0}\right)^{2}+\left(y-y_{0}\right)^{2}\right\rangle}{4 t}$,

where $x_{0}$ and $y_{0}$ are the initial co-ordinates of the particle, $x$ and $y$ its co-ordinates at time $t>>t_{p}, t_{p}=\frac{m}{\varsigma}$ is the velocity relaxation time, averaging is performed over the ensemble of particles. The value of diffusion coefficient obtained from simulation results was in the very good agreement with the theoretical value

$D_{0}=\frac{k T}{6 \pi \mu a}$.

The mean value of kinetic energy per particle remained in the interval 0.98$1.2 \mathrm{kT}$ during all simulations performed.

The residence time of particle in potential well was obtained in the following way. Two particles were placed initially at the distance corresponding to the minimum of the potential well and simulation of their relative motion was performed until the instant, when the distance between particles exceeded the range of colloidal interaction, $h_{2}$. The mean value of residence time was obtained by averaging over the results of 20 independent simulations. This mean value was then compared with the mean time of escape of particle from corresponding potential well calculated on the base of Smoluchowski equation [30] as (see Appendix):

$\bar{t}_{R}=\frac{1}{j}=\frac{\left(\int_{0}^{h_{2}} \exp \left(-\frac{U(x)}{k T}\right) d x \int_{x}^{h_{2}} \varsigma_{11}\left(x^{\prime}\right) \exp \left(\frac{U\left(x^{\prime}\right)}{k T}\right) d x^{\prime}\right)}{k T}$.

Comparison was performed for the values $U_{\min }=1,3,5$ and $7 \mathrm{kT}$ and have shown good agreement between the theory predictions and the simulations results (Table 1).

Table 1

\begin{tabular}{|l|c|c|c|c|}
\hline The potential well depth, kT & 1 & 3 & 5 & 7 \\
\hline $\begin{array}{l}\text { The mean residence time calculated } \\
\text { according to Eq.(18), s }\end{array}$ & 0.044 & 0.12 & 0.50 & 2.7 \\
\hline $\begin{array}{l}\text { The mean residence time obtained } \\
\text { by direct computer simulations, s }\end{array}$ & 0.024 & 0.11 & 0.46 & 2.4 \\
\hline The mean doublet life time, s & 0.36 & 0.65 & 4.0 & 13.9 \\
\hline
\end{tabular}


This value of the mean residence time of particle in potential well of course differs from the life time of doublet which could be observed in experiment, because particle can return back into the potential well several times until the separation becomes large enough to be detectable in experiment (Fig. 2). The last line in Table 1 shows for comparison the mean doublet life time, which is calculated as corresponding to the separation between particles $0.2 \mu \mathrm{m}$. After that particle can be considered as free moving particle according to our simulation results. Such a choice of doublet life time is an arbitrary one, however, it shows that the doublet life time is about one order of magnitude larger, than the residence time of a particle in the potential well.

For further verification of the model used let us consider the aggregation process in the deep enough potential well with $U_{\min }=10 \mathrm{kT}$. In this case the mean residence time determined according to Eq. (18) is about $38 \mathrm{~s}$. The latter means that we should expect situation close to the irreversible coagulation, especially in concentrated enough suspension, where the probability of interparticles collision is high and taking into account, that each particle in cluster is usually bounded to more than one particle.

Considered system is composed of 9 particles with radius $1 \mu \mathrm{m}$ placed in box of $5 \times 5$ mesh providing the solid 2D fraction of about 0.27 . To decrease the simulation time, the particles are placed close to each other, but still at the separations exceeding the range of colloidal forces. Only one particle is placed separately. The initial particles distribution is given in Fig. 3a. In Fig. 3 particles are shown in the box of 7x7 mesh. Additional meshes are added for convenience of presentation.

After the simulation started, some particles were separated due to action of Brownian forces: after $0.5 \mathrm{~s}$ of simulation there were two singlets, one doublet and one cluster with 5 particles. As colloidal interactions between particles in clusters are strong enough. Particles remained inside the clusters but they were moving inside clusters during the further evolution of the system changing the shape of the clusters. Further, the doublet aggregated with the bigger cluster building a large cluster with 7 particles. However, 2 singlets still remained free (Fig. 3c).

After $8 \mathrm{~s}$ one of the remaining singlets joined the cluster (Fig. 3d). Fig. 3d shows that last singlet was still in a free motion. Only after $18 \mathrm{~s}$ the last singlet joined 
the big cluster (Fig. 3e). After that particles were moving inside the cluster changing its shape. It seems that rod-like structure, presented in Fig. $3 \mathrm{f}$ is the most stable cluster shape in the case under consideration, however the question about preferable shape of clusters requires, of course more comprehensive study.

Further simulations were performed for an ensemble of 170 particles located in the simulation box of 20x20 mesh, solid 2D fraction around 0.31 . The mesh size was chosen in such a way that separation between two particles situated in the centres of adjacent mesh boxes was larger than range of colloidal forces. Two initial configuration of particles were used in simulations: (i) particles randomly distributed over the simulation box (Fig. 4a), (ii) particles (in this case 169 instead of 170) uniformly distributed over the simulation box (Fig. 5a). Simulations were performed with $h_{1}=1.6 \cdot 10^{-6} \mathrm{~cm}, h_{2}=3.0 \cdot 10^{-6} \mathrm{~cm}, h_{0}=2.0 \cdot 10^{-6} \mathrm{~cm}$ for the configuration (i), and with $h_{1}=1.6 \cdot 10^{-6} \mathrm{~cm}, h_{2}=1.0 \cdot 10^{-5} \mathrm{~cm}, h_{0}=2.0 \cdot 10^{-6} \mathrm{~cm}$ for the configuration (ii). The mesh size was chosen slightly smaller for configuration (ii), to keep nearly the same solid 2D fraction for both cases. Simulations were performed for $U_{\min }=3,6$ and $20 \mathrm{kT}$, what corresponds to mean residence time of particle in the potential well (i) of 0.12 , 1.14 and $4.5 \cdot 10^{5} \mathrm{~s}$ and in potential well (ii) of $0.50,3.95$ and $1.03 \cdot 10^{6} \mathrm{~s}$ according to Eq. (18). The mean separation between particles in the both model suspensions was around $l=1.6 \cdot 10^{-4} \mathrm{~cm}$. The mean diffusion coefficient of free particle according to Eq. (17) is about $D=2.2 \cdot 10^{-9} \mathrm{~cm}^{2} / \mathrm{s}$. The latter allows calculating the mean time between particles collision $\tau=l^{2} / 4 D$, which is about $2.6 \mathrm{~s}$. Note, hydrodynamic interaction as well as accounting for efficiency of collisions should result in an increase of this time. Therefore, the time required for doublet formation is substantially larger than life time of doublets at $U_{\min }=3 k T$. Those times are of the same order of magnitude at $U_{\min }=6 \mathrm{kT}$. However, the time required for doublet formation is much smallet than doublet life time at $U_{\min }=20 \mathrm{kT}$. Hence, it can be expected that a suspension with $U_{\min }=3 k T$ at equilibrium will be composed by small amount of doublets at the equilibrium with singlets. The probability the formation of larger clusters in this suspension is small. In a suspension with $U_{\min }=6 \mathrm{k} T$ the formation of triplets and even larger clusters can be expected but again at the equilibrium with singlets. In suspension with $U_{\min }=20 \mathrm{kT}$ the singlets should be absent at the equilibrium, whereas formation of rather large clusters is expected. Suspension considered in the case (ii) is 
expected to be less stable than suspension (i) because the residence time of particle in potential well is much larger for suspension (ii).

At the initial random particles distribution (Fig. 4a) the local solid volume fraction varies over the simulation box. Inside the areas with higher particles concentration the particles will coagulated faster than in other areas. Note, the depth of the potential well is crucial for the size of clusters formed (see Fig. 4). At $U_{\min }=20 \mathrm{kT}$ the large clusters are formed, the largest of them include 18 particles (Fig. 4b). The clustering process occurs very quickly at the beginning of the process but then it slows down (Fig. 6, curve 1), because still remaining singlets are at large separations from the clusters (Fig. 4b). At $U_{\min }=6 \mathrm{kT}$ the clusters are smaller (Fig. 4c) and the mean cluster size increases more slowly (Fig. 6. curve 2). At $U_{\min }=3 k T$ the clusters are mainly doublets and triplets (Fig. 4d), the mean cluster size even decreases with time (Fig. 6, curve3). The last is because the initially more intensive clustering occurred in the parts of the simulation box with larger local solid fraction.

The same trends are observed for the uniform initial distribution of particles (Fig. 5, Fig. 7). In this case, however, aggregation begins after an induction period, as particles initial separations in this case are larger, than separation in regions with larger local solid fraction in the case of the random distribution. The dependencies of mean number of particles in cluster on time (Fig. 7) at $U_{\min }=6 \mathrm{kT}$ and $U_{\min }=20 \mathrm{kT}$ almost coincide in the case of random initial distribution. The latter is because the time of simulations in the case of uniform initial distribution is essentially smaller as compared with the doublet lifetime estimated above.

\section{Conclusions}

The computer simulations based on the Langevin equation with colloidal interactions between particles and lubrication approximation for hydrodynamic interactions is used to perform the direct computer simulation of the processes of reversible aggregation of colloidal suspensions. Simulations allow predicting the correct value of diffusion coefficient of freely moving particle, the mean value of kinetic energy for each particle in ensemble of interacting colloidal particles and the residence times of colloidal particles inside the potential wells of different depths.

The computer simulations performed using the proposed model enabled the monitoring of formation and breakage of clusters in a suspension caused by competing colloidal interactions and thermal particle motion. It was shown, that at 
small depth of potential well ( $3 \mathrm{kT}$ ) the mean cluster size is less than 2, i.e. in this case small clusters coexist with singlets. An increase of the depth of the potential well results in the increase of the cluster size.

\section{Acknowledgement}

The authors would like to acknowledge the support from The Engineering and Physical Sciences Research Council, UK (Grant EP/C528557/1).

\section{Appendix}

Let us consider one particle in the potential well. Then according to the Smoluchowski equation the flux of particles in the field of force $F(x)=-d U / d x$ is [30]:

$$
j=\frac{1}{\varsigma_{11}} F w-D \frac{d w}{d x}=-\frac{1}{\varsigma_{11}} \frac{d U}{d x} w-\frac{k T}{\varsigma_{11}} \frac{d w}{d x},
$$

where $j$ is the particles steady state flux, $\mathrm{s}^{-1}, w(x)$ is the probability to find particle in the position $x, \mathrm{~cm}^{-1}, D$ is the particles diffusion coefficient, $\varsigma_{11}$ is the hydrodynamic resistance coefficient (see Eq (7) and (9)).

Rewriting the latter equation results in

$$
\frac{d w}{d x}+\frac{1}{k T} \frac{d U}{d x} w=-j \frac{\varsigma}{k T} .
$$

The solution of Eq. (A2) is tried in the following form:

$$
w=A(x) \exp \left(-\frac{U}{k T}\right)
$$

where $A(X)$ is a new unknown function. After substitution of expression (A3) into Eq. (A2) we obtain at steady state $(\mathrm{j}=$ const)

$$
A(x)=A_{0}-j \frac{1}{k T} \int_{0}^{x} \varsigma\left(x^{\prime}\right) \exp \left(\frac{U\left(x^{\prime}\right)}{k T}\right) d x^{\prime}
$$

and

$$
w(x)=A_{0} \exp \left(-\frac{U(x)}{k T}\right)-\frac{j}{k T} \exp \left(-\frac{U(x)}{k T}\right) \int_{0}^{x} \varsigma\left(x^{\prime}\right) \exp \left(\frac{U\left(x^{\prime}\right)}{k T}\right) d x^{\prime} .
$$

We are looking for a flux of a particle from the potential well in statistical sense, supposing that there is 1 particle in the potential well and no particles outside the well. The latter means that the following boundary conditions should be imposed 
$w\left(h_{2}\right)=0$,

$\int_{0}^{h_{2}} w(x) d x=1$

Using the condition (A6) we find

$$
\begin{aligned}
& A_{0}=\frac{j}{k T} \int_{0}^{h_{2}} \varsigma\left(x^{\prime}\right) \exp \left(\frac{U\left(x^{\prime}\right)}{k T}\right) d x^{\prime}, \\
& w(x)=\frac{j}{k T} \exp \left(-\frac{U(x)}{k T}\right) \int_{h}^{h_{2}} \varsigma\left(x^{\prime}\right) \exp \left(\frac{U\left(x^{\prime}\right)}{k T}\right) d x^{\prime}
\end{aligned}
$$

Substituting Eq. (A9) into Eq. (A7) we obtain

$$
1=\frac{j}{k T}\left(\int_{0}^{h_{2}} \exp \left(-\frac{U(x)}{k T}\right) d x \int_{x}^{h_{2}} \varsigma\left(x^{\prime}\right) \exp \left(\frac{U\left(x^{\prime}\right)}{k T}\right) d x^{\prime}\right)
$$

and

$$
j=\frac{k T}{\left(\int_{0}^{h_{2}} \exp \left(-\frac{U(x)}{k T}\right) d x \int_{x}^{h_{2}} \varsigma\left(x^{\prime}\right) \exp \left(\frac{U\left(x^{\prime}\right)}{k T}\right) d x^{\prime}\right)}
$$

$U(x)$ is given by :

$U(x)=\frac{U_{\min }}{R}\left[\frac{\left(h_{1}-x\right)^{3}}{3\left(h_{1}-h_{0}\right)}+\left(h_{2}-h_{1}\right)\left(h_{0}-x\right)+\frac{\left(h_{2}-h_{0}\right)^{2}-\left(h_{1}-h_{0}\right)^{2}}{3}\right]$ at $0 \leq \mathrm{x} \leq \mathrm{h}_{0}$

$$
U(x)=\frac{U_{\min }}{3 R} \frac{\left(h_{2}-x\right)^{3}}{3\left(h_{2}-h_{0}\right)}, \quad \text { at } \mathrm{h}_{0} \leq \mathrm{x} \leq \mathrm{h}_{2},
$$

where

$$
R=\frac{S^{3}}{3\left(h_{1}-h_{0}\right)}+\frac{\left(h_{2}-h_{0}\right)^{2}-\left(h_{1}-h_{0}\right)^{2}}{3}+\left(h_{2}-h_{1}\right)\left(S+h_{0}-h_{1}\right),
$$

and

$S=\sqrt{\left(h_{0}-h_{1}\right)\left(h_{2}-h_{1}\right)}$.

See Figs. $1 \mathrm{a}$ and $1 \mathrm{~b}$ for definitions of $\mathrm{h}_{0}, \mathrm{~h}_{1}, \mathrm{~h}_{2}$ and $\mathrm{U}_{\min }$. 


\section{References}

1. Deryaguin, B.V., Churaev, N.V., and Muller, V.M. "Surface forces", Consultants Bureau, Plenum Press, New York, 1987.

2. Israelachvili J.N. "Intermolecular and surface forces", Academic Press, London, 1992.

3. Long J.A., Osmond D.W.J., Vincent B. The equilibrium aspects of weak flocculation. J. Coll. Int. Sci. 1973, 42, 545.

4. Muller V.M. The theory of reversible coagulation. Colloid J. 1996, 58, 598.

5. Cornell R.M., Goodwin J.W., Ottewill R.H. Direct microscopic studies of particle motion in stable dispersions and in Floccules. J. Coll. Int. Sci. 1979, 71, 254.

6. Jeffrey G.C., Ottewill R.H. Reversible aggregation. Part 2. Kinetics of reversible aggregation. Colloid Polym. Sci. 1990, 268, 179.

7. Golikova E.V., Chernoberezhskii Yu.M., Ioganson O.M., Vysokovskaya N.A., Grigor'ev V.S. Stability of aqueous crystalline quartz dispersions in the acidic $\mathrm{pH}$ region: the role of the structural component of the particle interaction energy. Coll. J. 2003, 65, 420.

8. Segre P.N., Prasad V., Schofield A.B., Weitz D.A. Glasslike kinetic arrest at the colloidal-gelation transition. Phys. Rev. Letters, 2001, 86, 6042.

9. Dinsmore A.D., Weitz D.A. Direct imaging of three-demensional structure and topology of colloidal gels. J. Phys.: Condens. Matter, 2002, 14, 7581.

10. Sedgwick H., Egelhaaf S.U., and Poon, W.C.K. Clusters and gels in systems of sticky particles. J. Phys.: Condens. Matter, 2004, 16, S4913.

11. Stradner A., Sedgwick H., Cardinaux F., Poon, W.C.K., Egelhaaf S.U., Schurtenberger P. Equilibrium cluster formation in concentrated protein solutions and colloids. Nature, 2004, 432, 492.

12. Campbell A.I., Anderson V.J., van Duijneveldt J.S., and Bartlett P. Dynamical arrest in attractive colloids: the effect of long-range repulsion. Phys. Rev. Letters, 2005, 94, 208301.

13. Sanchez R., Bartlett P. Equilibrium cluster formation and gelation. J. Phys.: Condens. Matter, 2005, 17, S3551. 
14. Groenewold J., and Kegel W.K. Anomalously large equilibrium clusters of colloids. J. Phys. Chem. B, 2001, 105, 11702.

15. Sciortino F., Mossa S., Zaccareli E., and Tartaglia P. Equilibrium cluster phases and low-density arrested disordered states: the role of short-range attraction and long-range repulsion. Phys. Rev. Lett., 2004, 93, 055701.

16. Mossa S., Sciortino F., Tartaglia P., and Zaccareli E. Ground-state clusters for short-range attractive and long-range repulsive potentials. Langmuir, 2004, 20, 10756.

17. Victor J.M., Hansen J.P. Spinodal decomposition and the liquid-vapour equilibrium in charged colloidal dispersions. J. Chem. Soc. Faraday Trans. 2. 1985, 81, 43.

18. Kaldash J. Laven J. Stein H.N. Equilibrium phase diagram of suspensions of electrically stabilized colloidal particles. Langmuir, 1996, 12, 6197.

19. Lai S.K., Peng W.P., Wang G.F. Realistic calculation of the low- and highdensity liquid phase separation in a charged colloidal dispersion. Phys. Rev. E. 2001, 63, 041511

20. Lai S.K., Wu K.L. Liquid-liquid and liquid-solid phase separation and flocculation for a charged colloidal dispersion. Phys. Rev. E. 2002, 66, 041403.

21. Haw M.D., Sievwright M., Poon W.C.K, and Pusey P.N. Cluster-cluster gelation with finite bond energy. Adv. Coll. Int. Sci., 1995, 62, 1.

22. Odrizola G., Schmitt A., Moncho-Jorda A., Callejas-Fernandez J., MartinezGarcia R., Leone R., and Hidalgo-Alvarez R. Constant bond breakup probability model for reversible aggregation processes. Phys. Rev. E, 2002, 65, 031405.

23. Puertas A.M., and Odrizola G. Linking phase behaviour and reversible colloidal aggregation at low concentrations: simulations and stochastic mean field theory. J. Phys. Chem. B, 2007, 111, 5564.

24. Uriev N.B., Kuchin I.V. Modelling of flow processes of the structured twophase disperse systems (solid phase-liquid medium). Adv. Col. Int. Sci. 2007, 134-135, 249.

25. Coffey W.T., Kalmykov Yu.P., and Waldron J.T. "The Langevin equation. With applications in physics, chemistry and electrical engineering.” World 
scientific series in contemporary chemical physics. Vol. 11., World scientific publishing 1996.

26. Ermak D.L., and McCammon J.A. Brownian dynamics with hydrodynamic interactions. J. Chem. Phys., 1978, 69, 1352.

27. Cox R.G. The motion of suspended particles almost in contact. Int. J. Multiphase Flow, 1974, 1, 343.

28. Turq P., Lanteime F., and Friedman H.L. Brownian dynamic: its application to ionic solutions. J. Chem. Phys., 1977, 66, 3039.

29. Groot R.D., and Warren P.B. Dissipative particle dynamics: Bridging the gap between atomistic and mesoscopic simulation. J. Chem. Phys., 1997, 107, 4423.

30. Chandrasekhar S. Stochastic problems in physics and astronomy. Reviews Modern Physics, 1943, 15, 1. 


\section{Figure legends}

Fig. 1. Colloidal interaction between particles used in the computer simulations: a the normal force per unit area between two parallel flat surfaces, b - energy of interaction between particles: $h_{1}=1.6 \cdot 10^{-6} \mathrm{~cm}, h_{0}=2.0 \cdot 10^{-6} \mathrm{~cm}, h_{2}=3.0 \cdot 10^{-6} \mathrm{~cm}, 1-$ $U_{\min }=3 \mathrm{kT}, 2-U_{\min }=20 \mathrm{kT}$.

Fig. 2 Time dependence of distance between particles in doublet at $U_{\min }=5 \mathrm{kT}$.

Fig. 3. Aggregation of colloidal particles in potential well with $h_{1}=1.6 \cdot 10^{-6} \mathrm{~cm}$, $h_{0}=2.0 \cdot 10^{-6} \mathrm{~cm}, h_{2}=3.0 \cdot 10^{-6} \mathrm{~cm}, U_{\min }=10 \mathrm{kT}$. Solid 2D fraction 0.27 .

Fig. 4. Time evolution of ensemble of particles with initial random distribution. Parameters of potential well: $h_{1}=1.6 \cdot 10^{-6} \mathrm{~cm}, h_{0}=2.0 \cdot 10^{-6} \mathrm{~cm}, h_{2}=3.0 \cdot 10^{-6} \mathrm{~cm}$, a initial distribution, b $-U_{\min }=20 \mathrm{kT}, \mathrm{c}-U_{\min }=6 \mathrm{kT}, \mathrm{d}-U_{\min }=3 \mathrm{kT}$.

Fig. 5. Time evolution of ensemble of particles with initial uniform distribution. Parameters of potential well: $h_{1}=1.6 \cdot 10^{-6} \mathrm{~cm}, h_{0}=2.0 \cdot 10^{-6} \mathrm{~cm}, h_{2}=1.0 \cdot 10^{-5} \mathrm{~cm}$, a initial distribution, b $-U_{\min }=20 \mathrm{kT}, \mathrm{c}-U_{\min }=6 \mathrm{kT}, \mathrm{d}-U_{\min }=3 \mathrm{kT}$.

Fig. 6. Time dependence of the mean number of particles in cluster for the random initial distribution: $1-U_{\min }=20 \mathrm{kT}, 2-U_{\min }=6 \mathrm{kT}, 3-U_{\min }=3 \mathrm{kT}$.

Fig. 7. Time dependence of the mean number of particles in cluster for the uniform initial distribution: $1-U_{\min }=20 \mathrm{kT}, 2-U_{\min }=6 \mathrm{kT}, 3-U_{\min }=3 \mathrm{kT}$. 


\section{Figures}

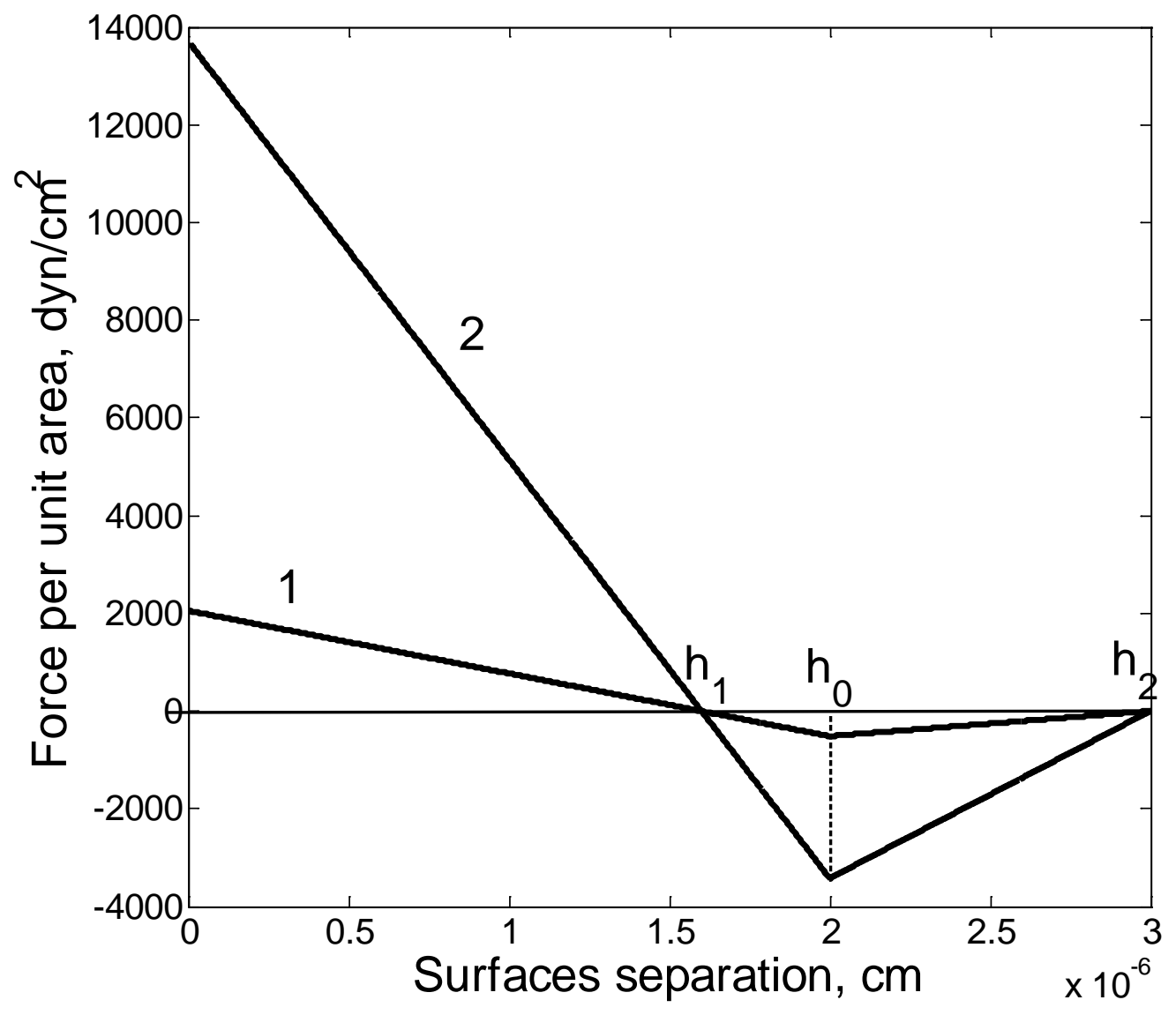

Fig. 1a

Fig. 1. Colloidal interaction between particles used in the computer simulations:

$\mathrm{a}$ - the normal force per unit area between two parallel flat surfaces,

b - energy of interaction between particles: $h_{1}=1.6 \cdot 10^{-6} \mathrm{~cm}, h_{0}=2.0 \cdot 10^{-6} \mathrm{~cm}$, $h_{2}=3.0 \cdot 10^{-6} \mathrm{~cm}, 1-U_{\min }=3 \mathrm{kT}, 2-U_{\min }=20 \mathrm{kT}$. 


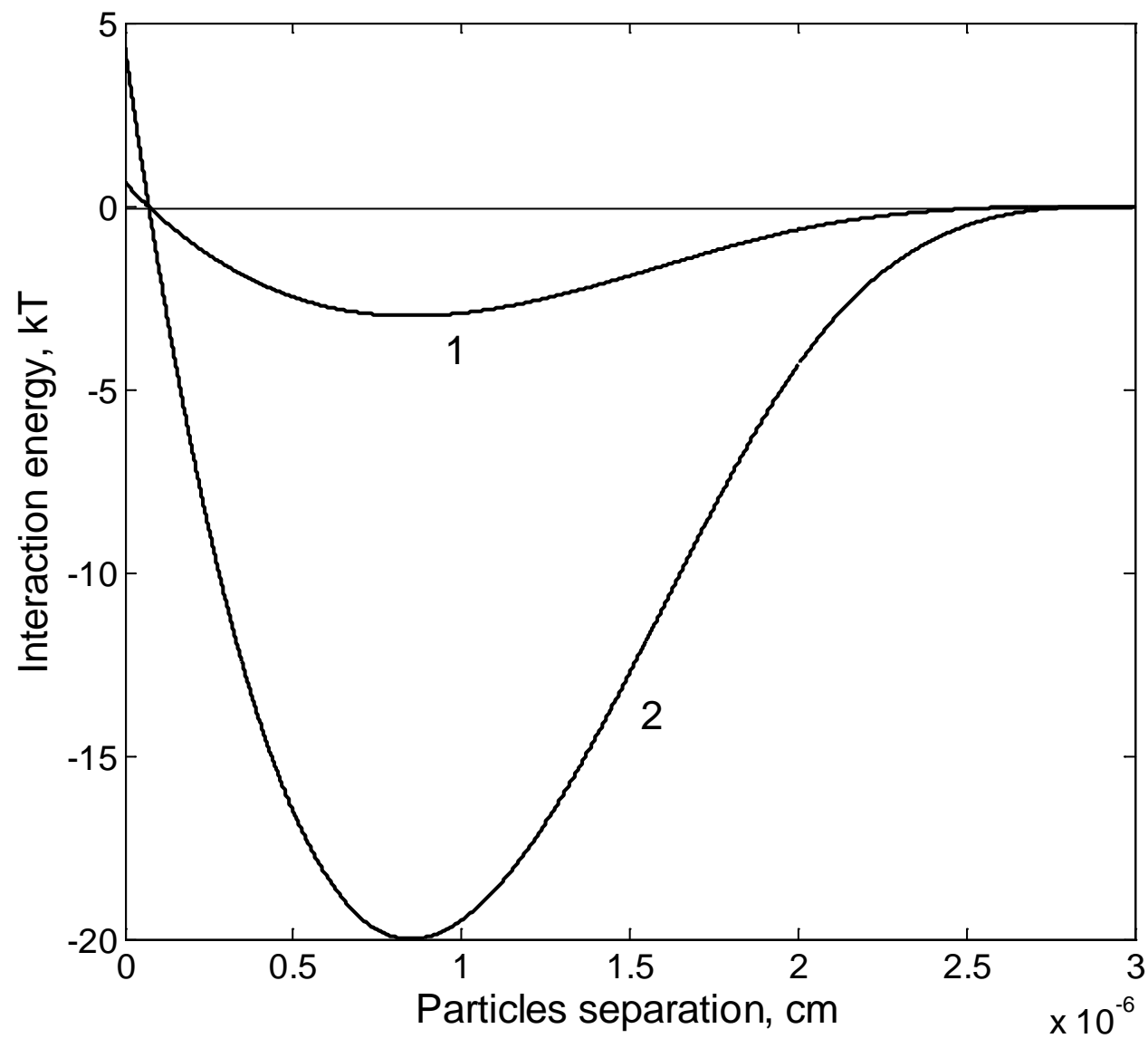

Fig. 1b

Fig. 1. Colloidal interaction between particles used in the computer simulations: a - the normal force per unit area between two parallel flat surfaces,, b - energy of interaction between particles: $h_{1}=1.6 \cdot 10^{-6} \mathrm{~cm}, h_{0}=2.0 \cdot 10^{-6} \mathrm{~cm}$, $h_{2}=3.0 \cdot 10^{-6} \mathrm{~cm}, 1-U_{\min }=3 \mathrm{kT}, 2-U_{\min }=20 \mathrm{kT}$. 


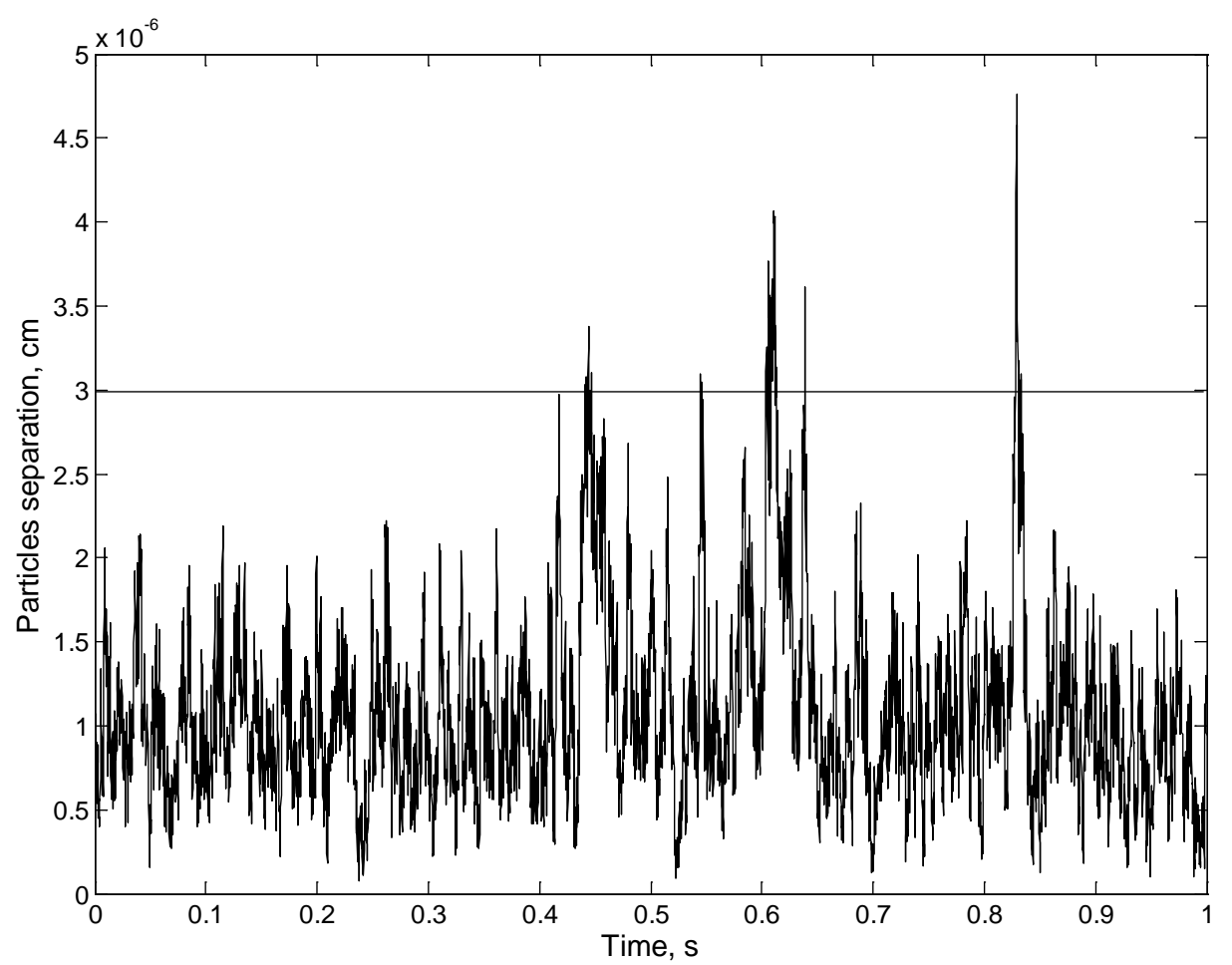

Fig. 2

Fig. 2 Time dependence of distance between particles in doublet at $U_{\min }=5 \mathrm{kT}$. 


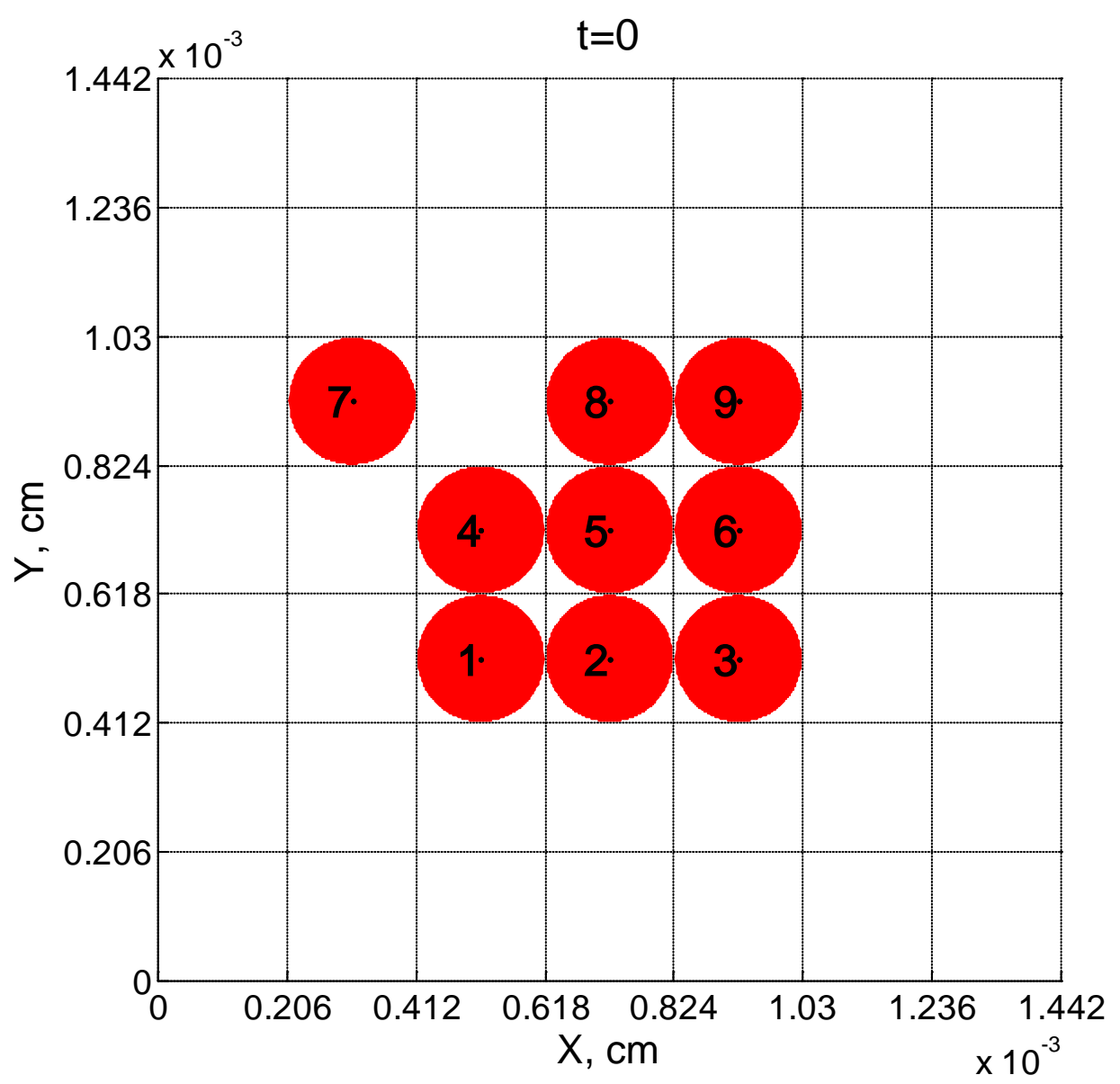

Fig. 3a

Fig. 3. Aggregation of colloidal particles in potential well with $h_{1}=1.6 \cdot 10^{-6} \mathrm{~cm}$, $h_{0}=2.0 \cdot 10^{-6} \mathrm{~cm}, h_{2}=3.0 \cdot 10^{-6} \mathrm{~cm}, U_{\min }=10 \mathrm{kT}$. Solid 2D fraction 0.27. Initial particle distribution. 


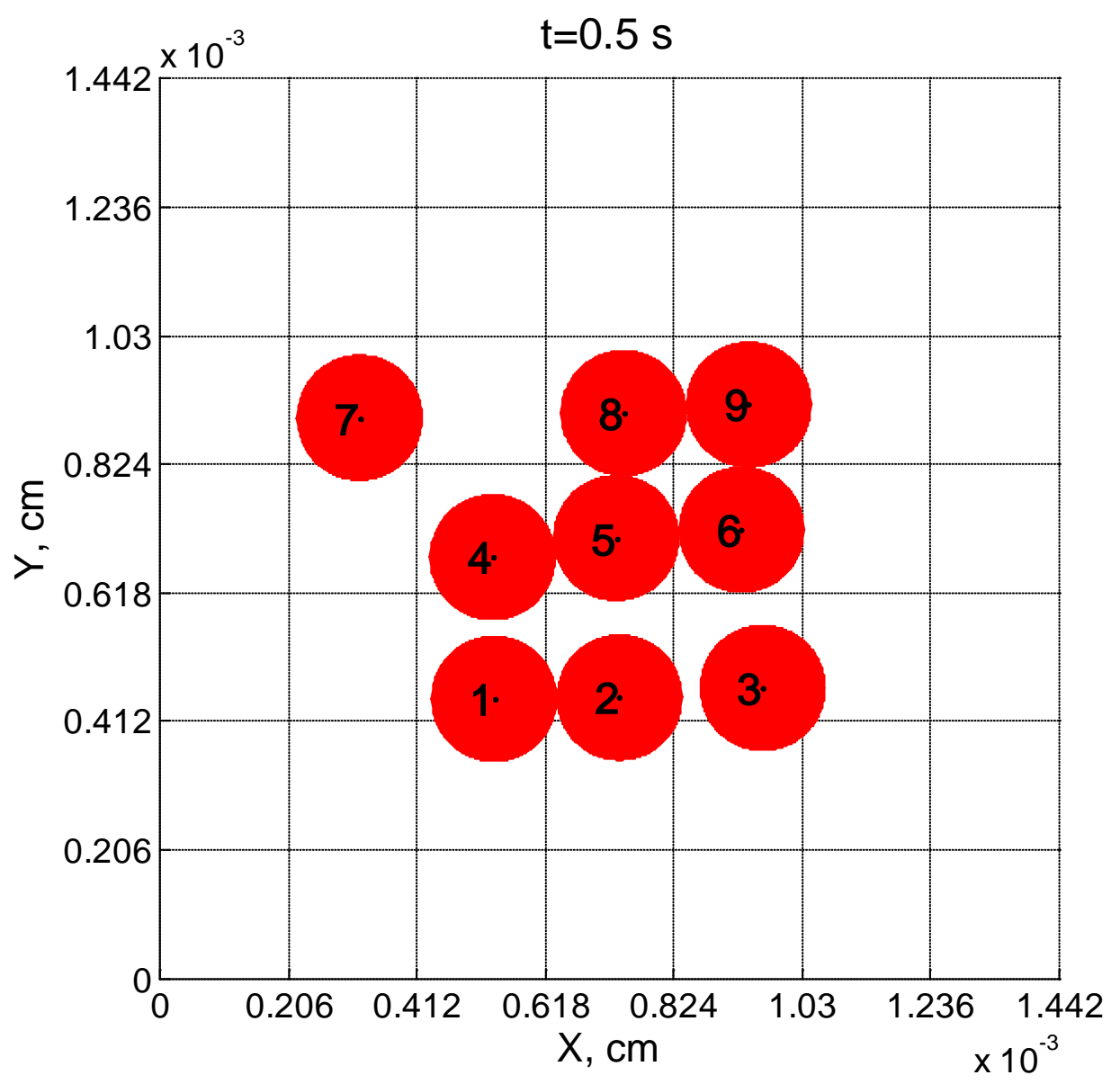

Fig. $3 b$

Fig. 3. Aggregation of colloidal particles in potential well with $h_{1}=1.6 \cdot 10^{-6} \mathrm{~cm}$, $h_{0}=2.0 \cdot 10^{-6} \mathrm{~cm}, h_{2}=3.0 \cdot 10^{-6} \mathrm{~cm}, U_{\min }=10 \mathrm{kT}$. Solid 2D fraction 0.27. particle distribution after $0.5 \mathrm{sec}$. 


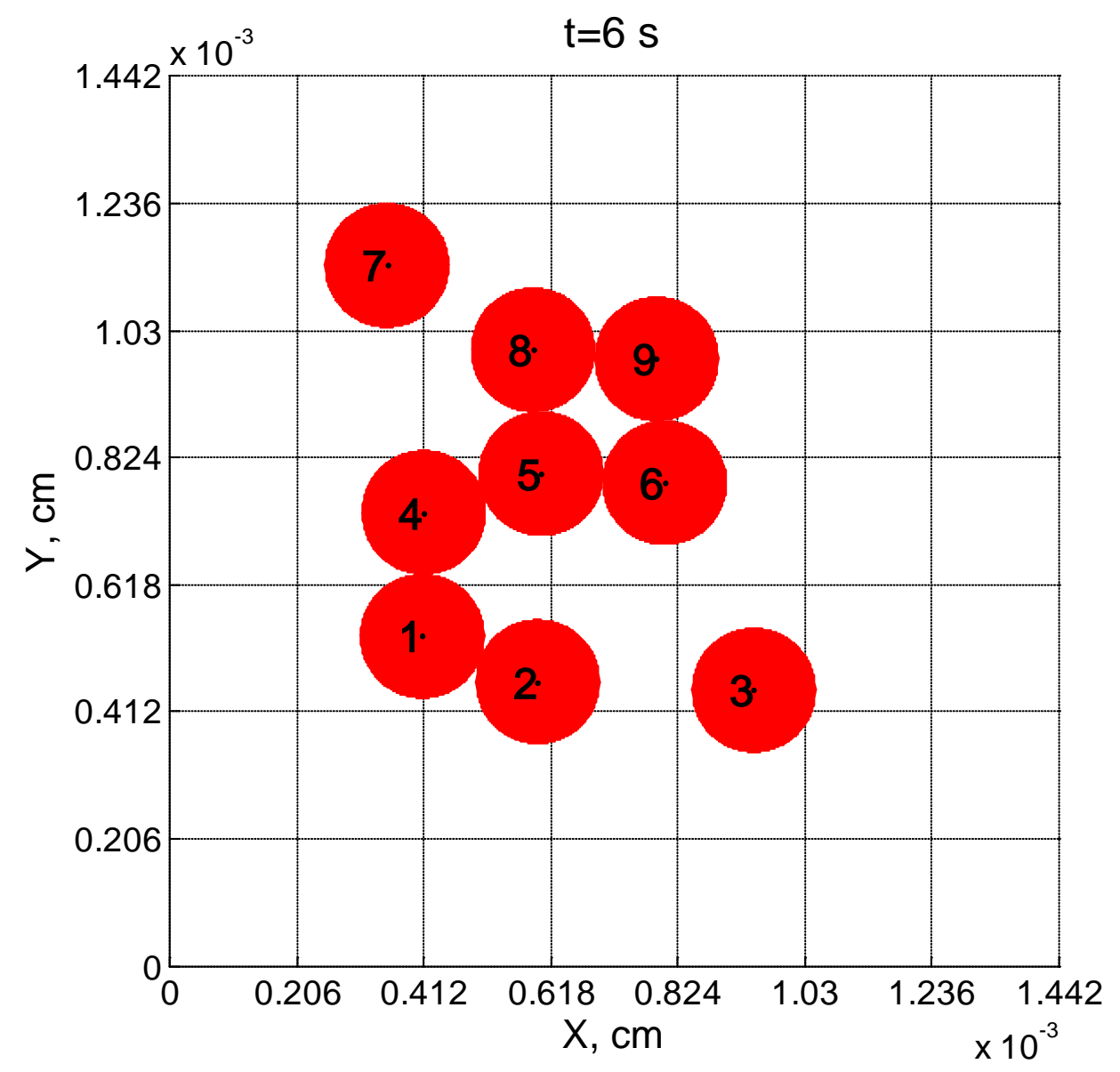

Fig. 3c

Fig. 3. Aggregation of colloidal particles in potential well with $h_{1}=1.6 \cdot 10^{-6} \mathrm{~cm}$, $h_{0}=2.0 \cdot 10^{-6} \mathrm{~cm}, h_{2}=3.0 \cdot 10^{-6} \mathrm{~cm}, U_{\min }=10 \mathrm{kT}$. Solid 2D fraction 0.27. Particle distribution after 6 sec. 


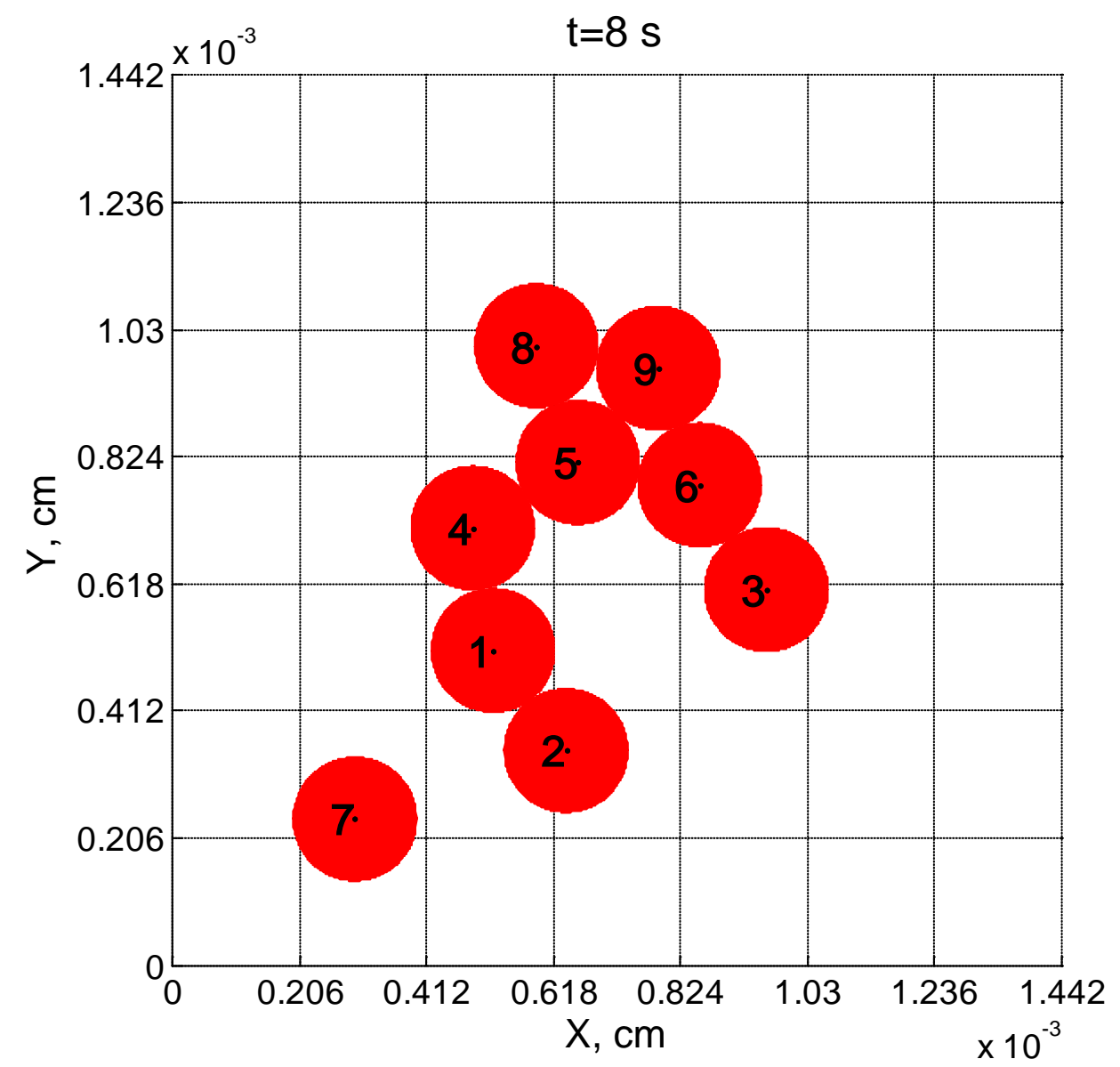

Fig. 3d

Fig. 3. Aggregation of colloidal particles in potential well with $h_{1}=1.6 \cdot 10^{-6} \mathrm{~cm}$, $h_{0}=2.0 \cdot 10^{-6} \mathrm{~cm}, h_{2}=3.0 \cdot 10^{-6} \mathrm{~cm}, U_{\min }=10 \mathrm{kT}$. Solid 2D fraction 0.27. Particle distribution after 8 sec. 


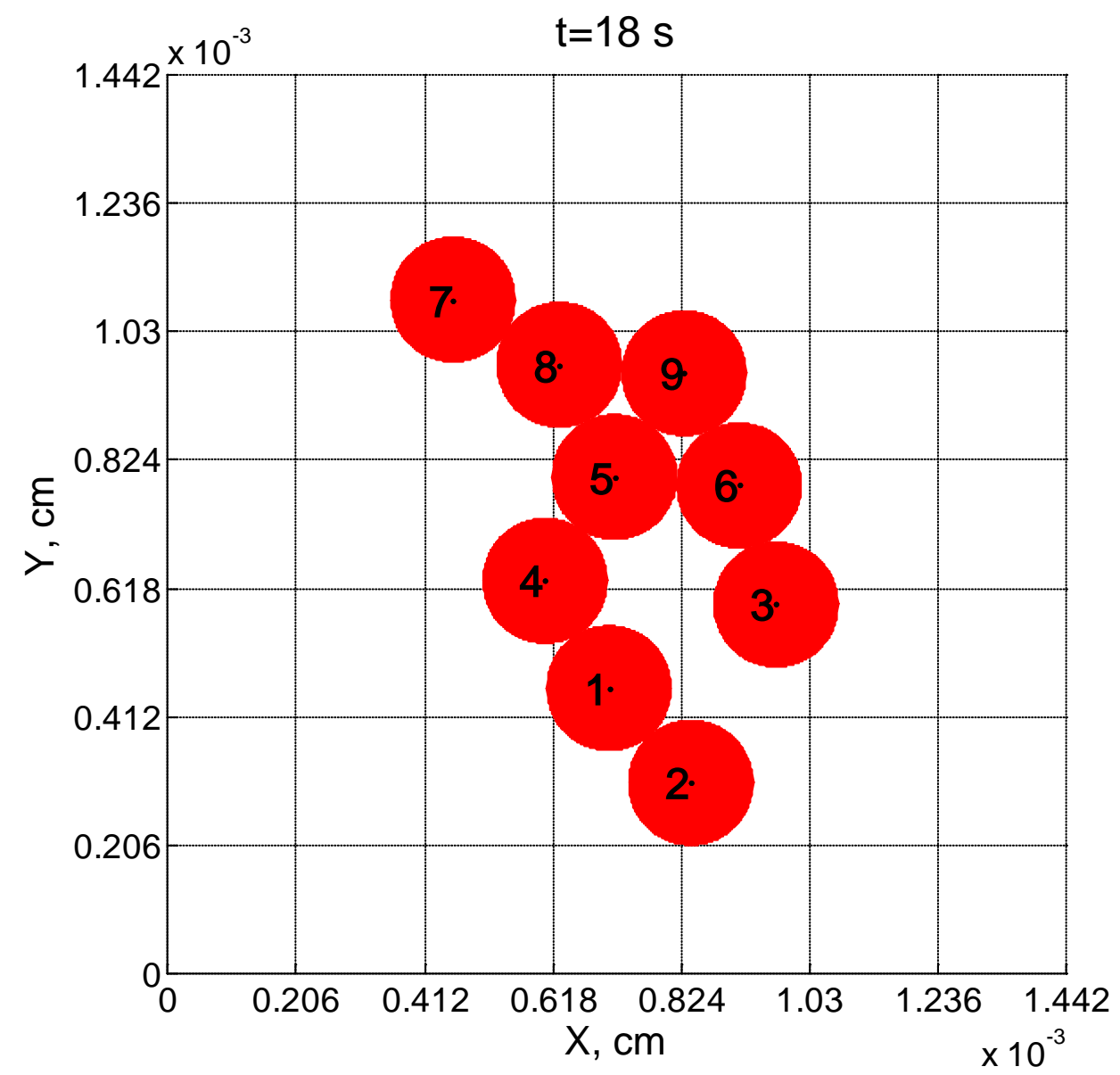

Fig. 3e

Fig. 3. Aggregation of colloidal particles in potential well with $h_{1}=1.6 \cdot 10^{-6} \mathrm{~cm}$, $h_{0}=2.0 \cdot 10^{-6} \mathrm{~cm}, h_{2}=3.0 \cdot 10^{-6} \mathrm{~cm}, U_{\min }=10 \mathrm{kT}$. Solid 2D fraction 0.27. Particle distribution after $18 \mathrm{sec}$. 


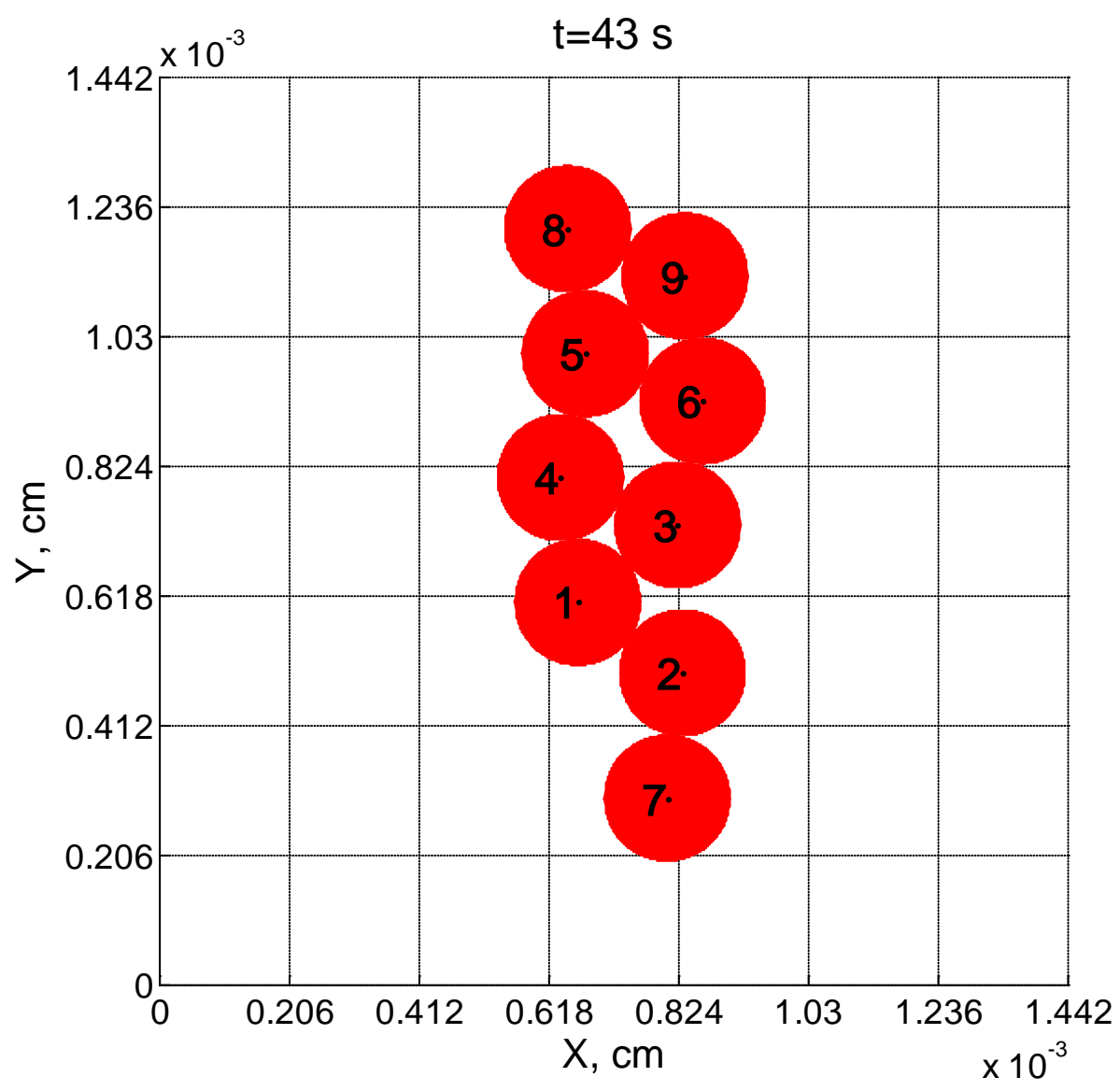

Fig. $3 f$

Fig. 3. Aggregation of colloidal particles in potential well with $h_{1}=1.6 \cdot 10^{-6} \mathrm{~cm}$, $h_{0}=2.0 \cdot 10^{-6} \mathrm{~cm}, h_{2}=3.0 \cdot 10^{-6} \mathrm{~cm}, U_{\min }=10 \mathrm{kT}$. Solid 2D fraction 0.27 . Particle distribution after $43 \mathrm{sec}$. 


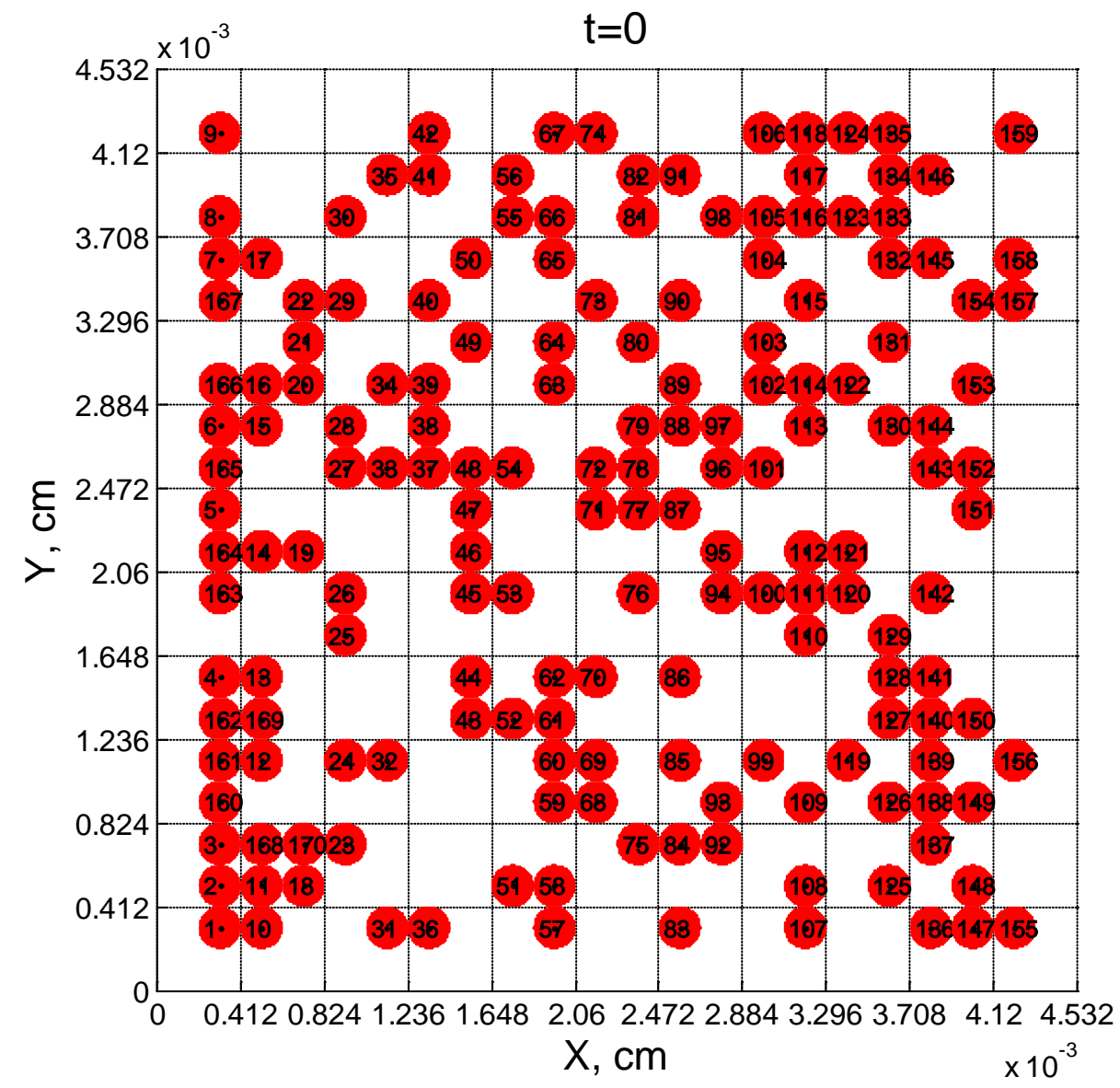

Fig. $4 \mathrm{a}$

Fig. 4. Time evolution of ensemble of particles with initial random distribution. Parameters of potential well: $h_{1}=1.6 \cdot 10^{-6} \mathrm{~cm}, h_{0}=2.0 \cdot 10^{-6} \mathrm{~cm}, h_{2}=3.0 \cdot 10^{-6} \mathrm{~cm}$, a initial distribution, b $-U_{\min }=20 \mathrm{kT}, \mathrm{c}-U_{\min }=6 \mathrm{kT}, \mathrm{d}-U_{\min }=3 \mathrm{kT}$. Initial particle distribution. 


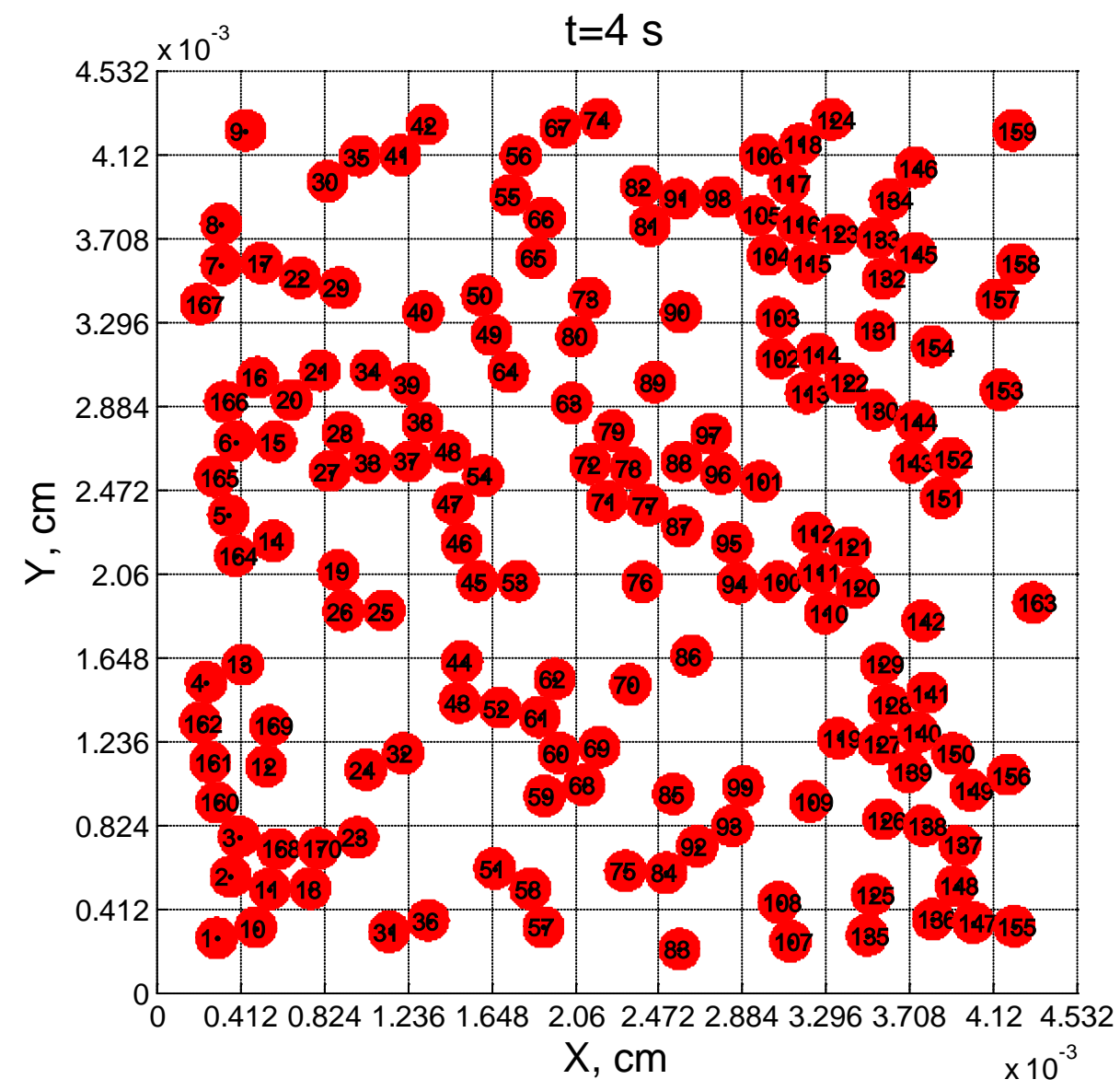

Fig. 4b

Fig. 4. Time evolution of ensemble of particles with initial random distribution. Parameters of potential well: $h_{1}=1.6 \cdot 10^{-6} \mathrm{~cm}, h_{0}=2.0 \cdot 10^{-6} \mathrm{~cm}, h_{2}=3.0 \cdot 10^{-6} \mathrm{~cm}, \mathrm{a}-$ initial distribution, b $-U_{\min }=20 \mathrm{kT}$, c $-U_{\min }=6 \mathrm{kT}, \mathrm{d}-U_{\min }=3 \mathrm{kT}$. Particle distribution after 4 sec. 


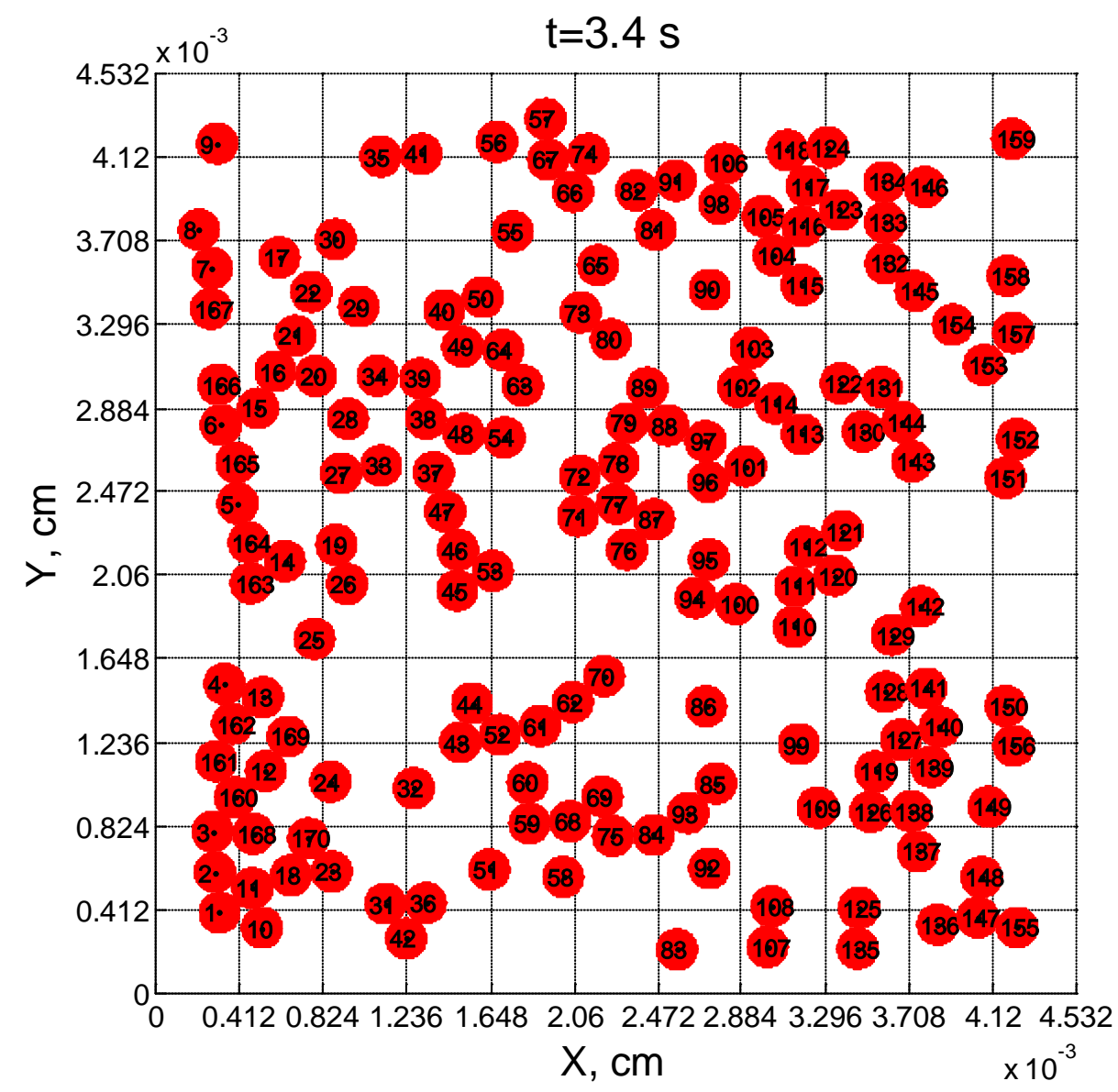

Fig. 4c

Fig. 4. Time evolution of ensemble of particles with initial random distribution. Parameters of potential well: $h_{1}=1.6 \cdot 10^{-6} \mathrm{~cm}, h_{0}=2.0 \cdot 10^{-6} \mathrm{~cm}, h_{2}=3.0 \cdot 10^{-6} \mathrm{~cm}$, a initial distribution, b $-U_{\min }=20 \mathrm{kT}$, c $-U_{\min }=6 \mathrm{kT}, \mathrm{d}-U_{\min }=3 \mathrm{kT}$. Particle distribution after 3.4 sec. 


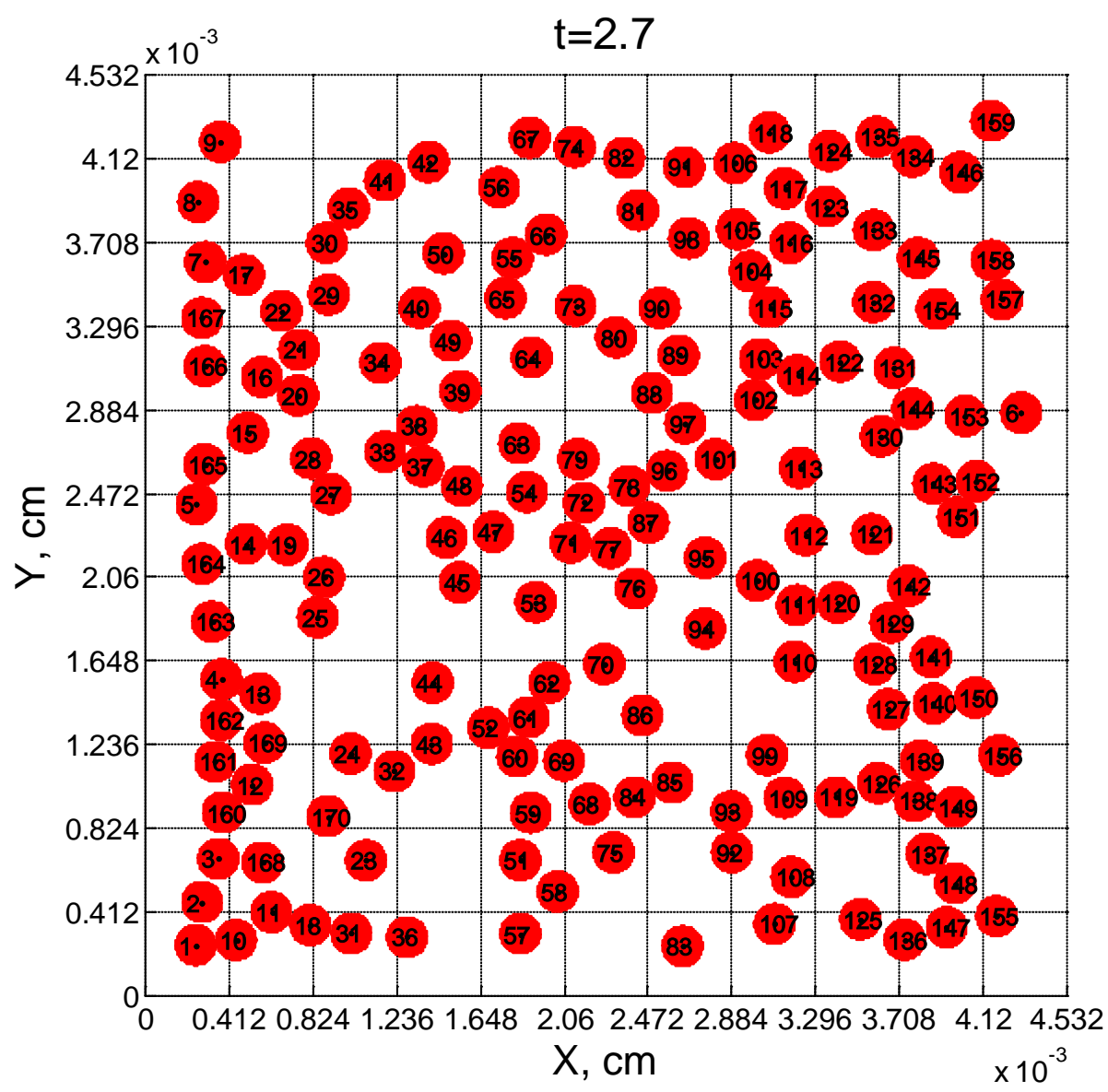

Fig. 4d

Fig. 4. Time evolution of ensemble of particles with initial random distribution. Parameters of potential well: $h_{1}=1.6 \cdot 10^{-6} \mathrm{~cm}, h_{0}=2.0 \cdot 10^{-6} \mathrm{~cm}, h_{2}=3.0 \cdot 10^{-6} \mathrm{~cm}$, a initial distribution, $\mathrm{b}-U_{\min }=20 \mathrm{kT}$, c $-U_{\min }=6 \mathrm{kT}, \mathrm{d}-U_{\min }=3 \mathrm{kT}$. Particle distribution after 2.7 sec. 


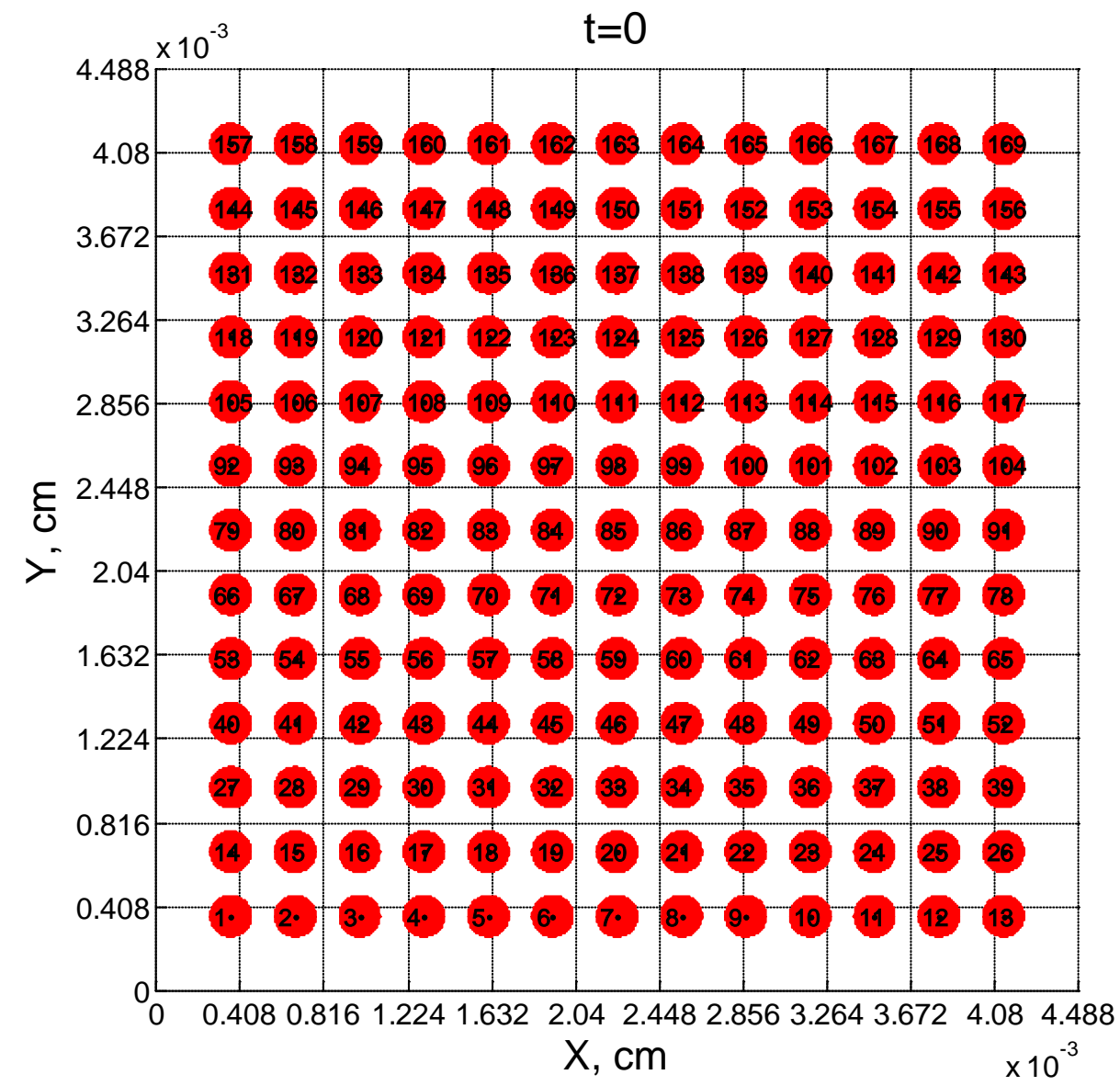

Fig. $5 \mathrm{a}$

Fig. 5. Time evolution of ensemble of particles with initial uniform distribution. Parameters of potential well: $h_{1}=1.6 \cdot 10^{-6} \mathrm{~cm}, h_{0}=2.0 \cdot 10^{-6} \mathrm{~cm}, h_{2}=1.0 \cdot 10^{-5} \mathrm{~cm}$, a initial distribution, b $-U_{\min }=20 \mathrm{kT}$, c $-U_{\min }=6 \mathrm{kT}$, d $-U_{\min }=3 \mathrm{kT}$. Initial particle distribution. 


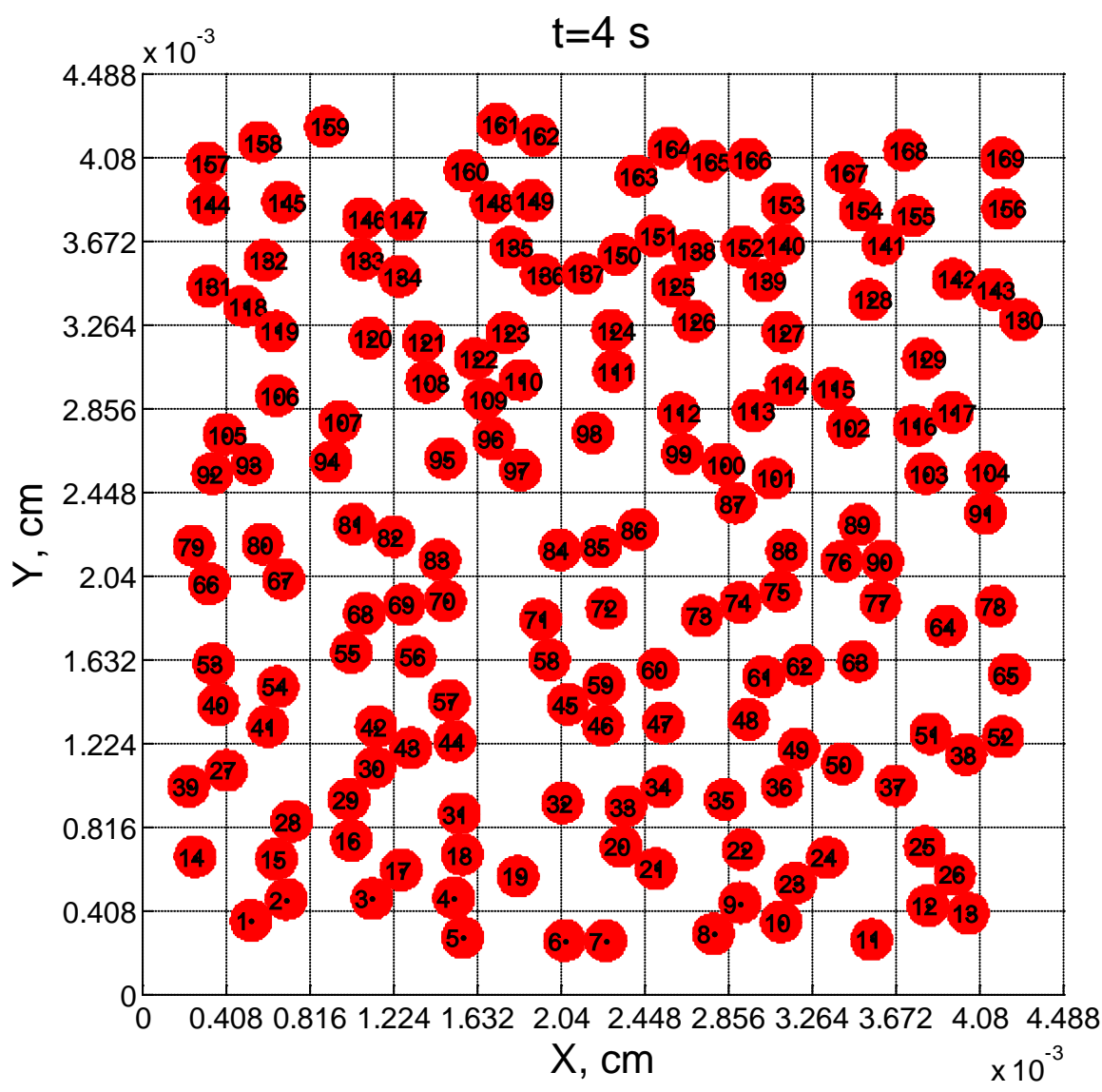

Fig. 5b

Fig. 5. Time evolution of ensemble of particles with initial uniform distribution. Parameters of potential well: $h_{1}=1.6 \cdot 10^{-6} \mathrm{~cm}, h_{0}=2.0 \cdot 10^{-6} \mathrm{~cm}, h_{2}=1.0 \cdot 10^{-5} \mathrm{~cm}$, a initial distribution, b $-U_{\min }=20 \mathrm{kT}$, c $-U_{\min }=6 \mathrm{kT}, \mathrm{d}-U_{\min }=3 \mathrm{kT}$. Particle distribution after 4 sec. 


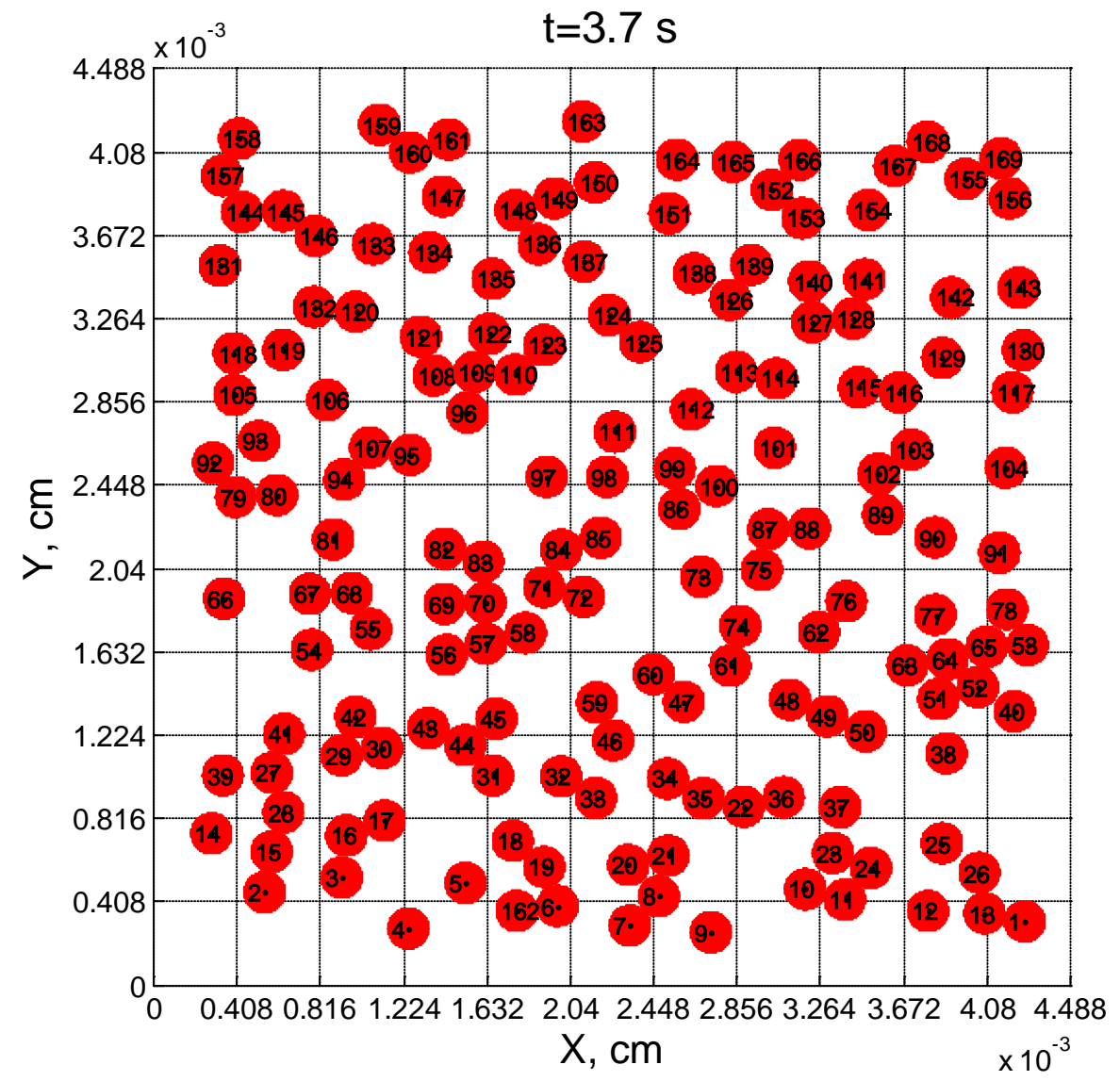

Fig. 5c

Fig. 5. Time evolution of ensemble of particles with initial uniform distribution. Parameters of potential well: $h_{1}=1.6 \cdot 10^{-6} \mathrm{~cm}, h_{0}=2.0 \cdot 10^{-6} \mathrm{~cm}, h_{2}=1.0 \cdot 10^{-5} \mathrm{~cm}$, a initial distribution, b $-U_{\min }=20 \mathrm{kT}$, c $-U_{\min }=6 \mathrm{kT}, \mathrm{d}-U_{\min }=3 \mathrm{kT}$. Particle distribution after 3.7 sec. 


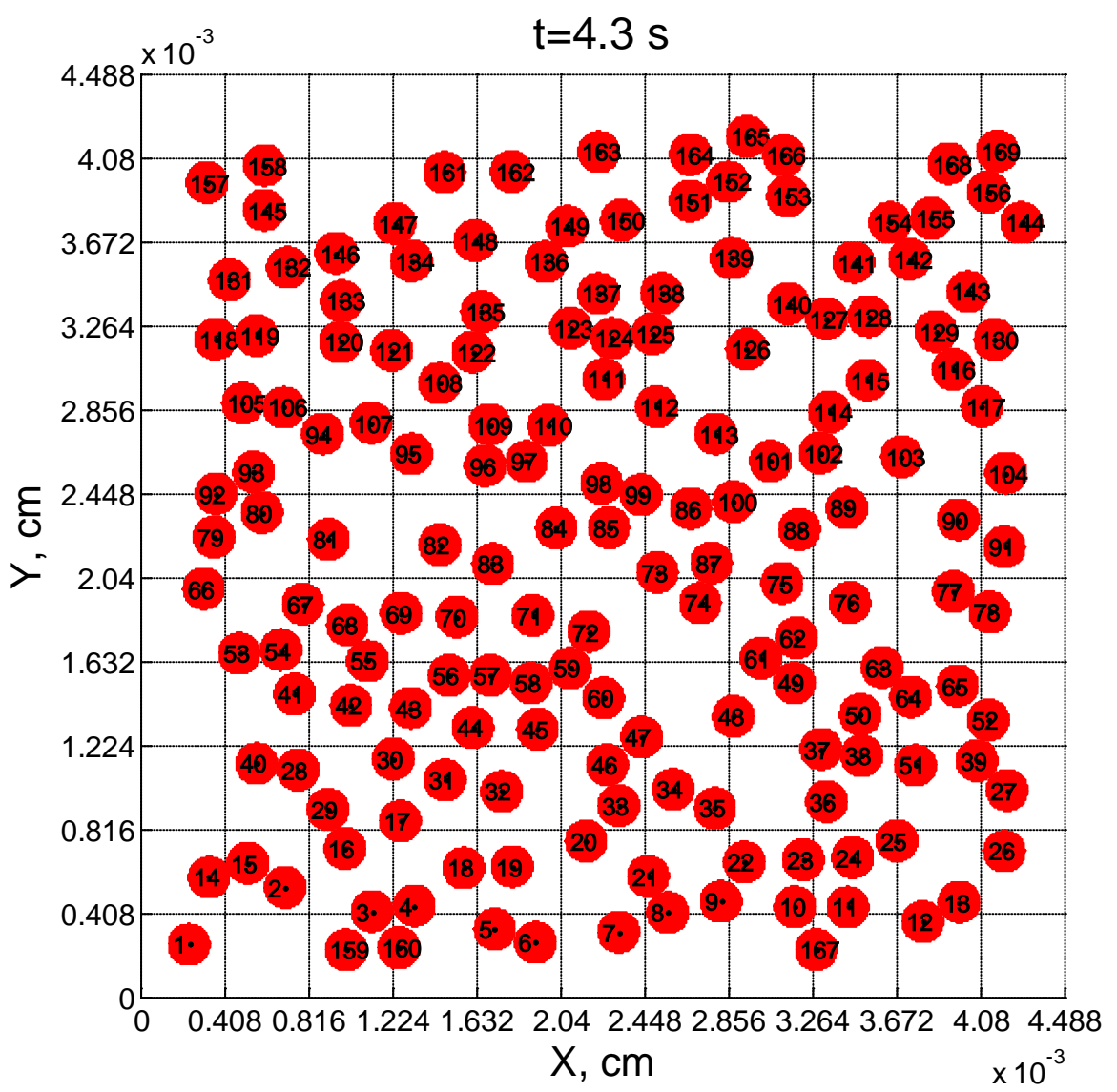

Fig. 5d

Fig. 5. Time evolution of ensemble of particles with initial uniform distribution. Parameters of potential well: $h_{1}=1.6 \cdot 10^{-6} \mathrm{~cm}, h_{0}=2.0 \cdot 10^{-6} \mathrm{~cm}, h_{2}=1.0 \cdot 10^{-5} \mathrm{~cm}$, a initial distribution, b $-U_{\min }=20 \mathrm{kT}$, c $-U_{\min }=6 \mathrm{kT}, \mathrm{d}-U_{\min }=3 \mathrm{kT}$. Particle distribution after 4.3 sec. 


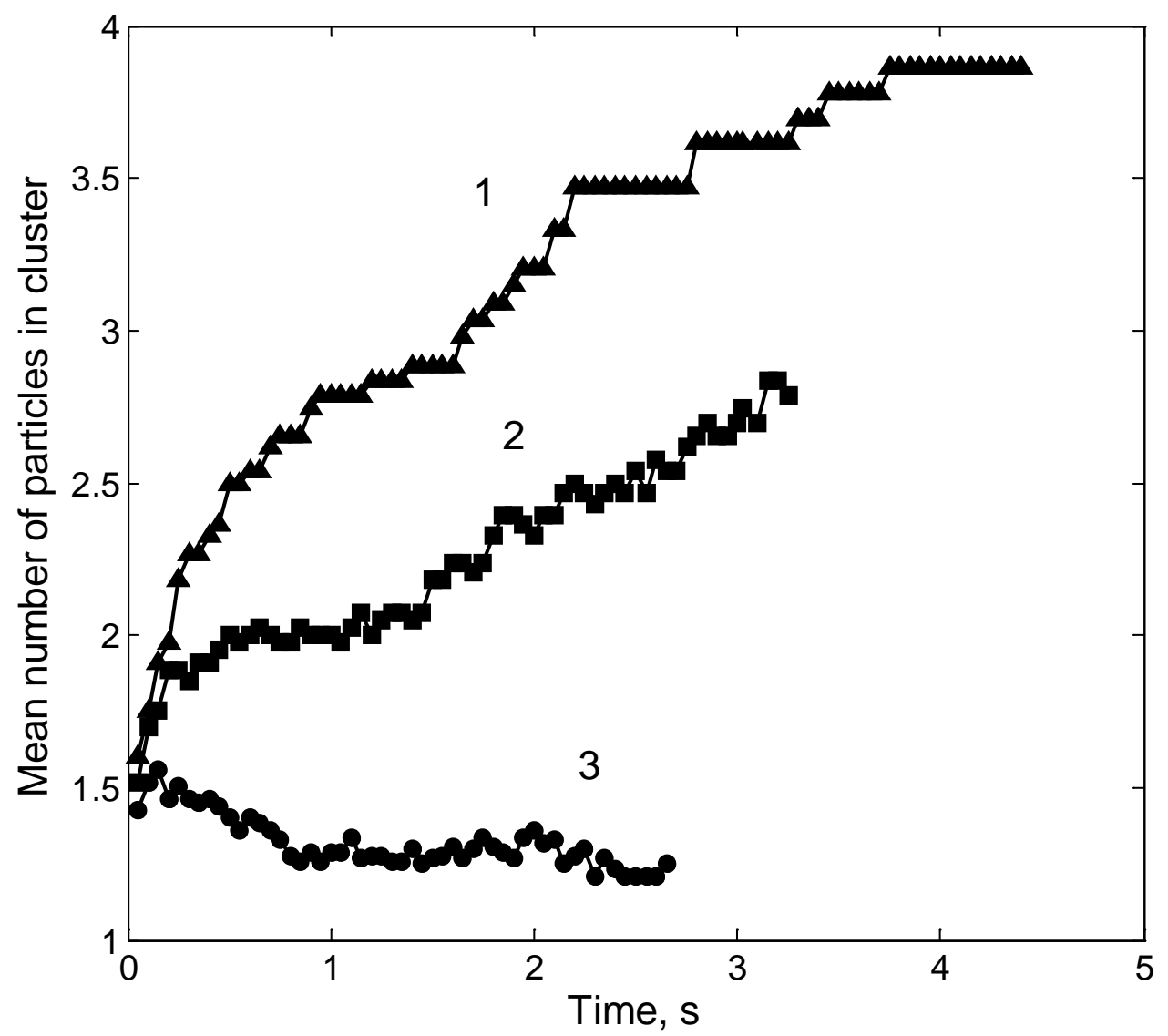

Fig. 6

Fig. 6. Time dependence of the mean number of particles in cluster for the random initial distribution: $1-U_{\min }=20 \mathrm{kT}, 2-U_{\min }=6 \mathrm{kT}, 3-U_{\min }=3 \mathrm{kT}$. 


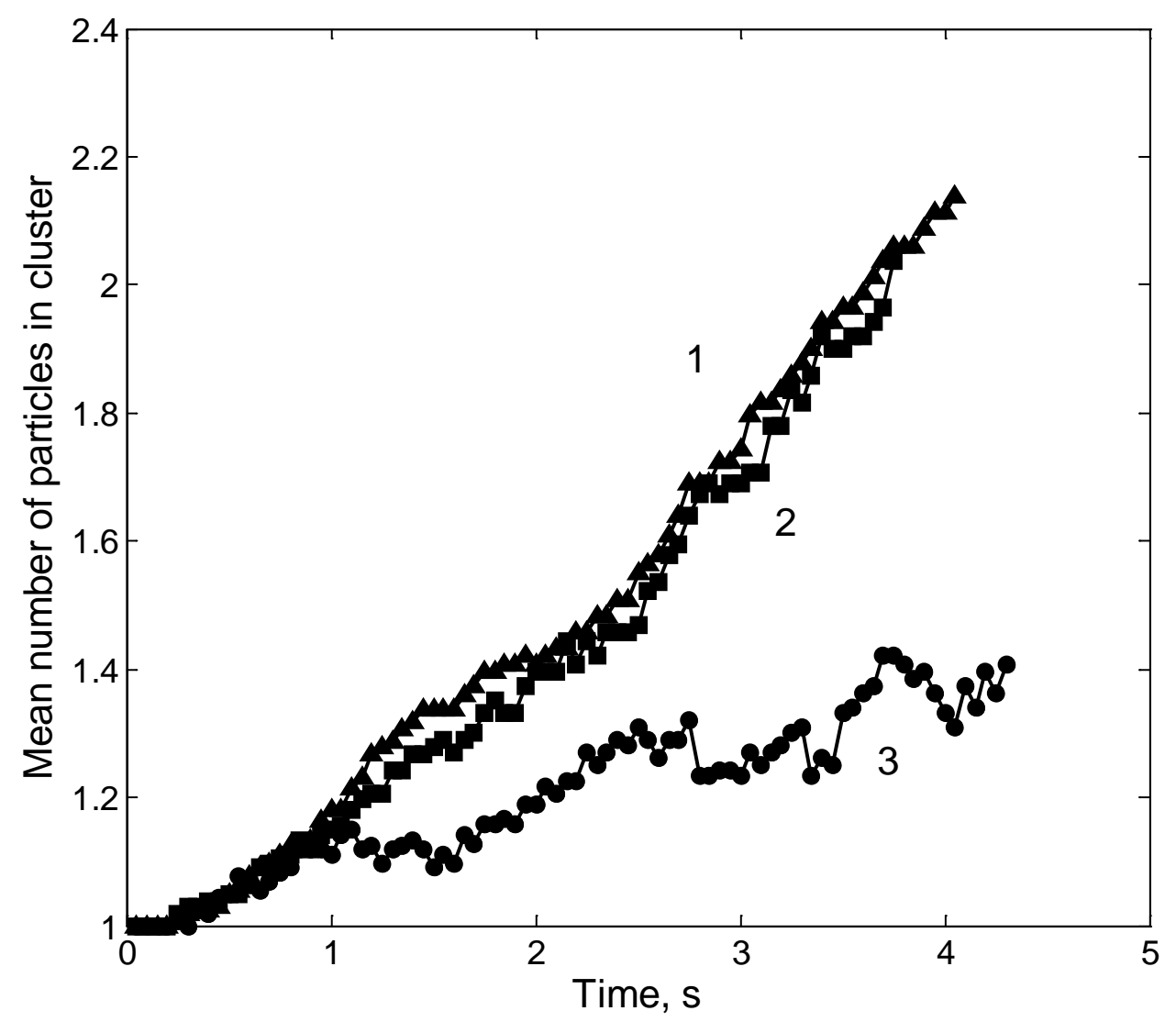

Fig. 7

Fig. 7. Time dependence of the mean number of particles in cluster for the uniform initial distribution: $1-U_{\min }=20 \mathrm{kT}, 2-U_{\min }=6 \mathrm{kT}, 3-U_{\min }=3 \mathrm{kT}$. 